\title{
A CLASS OF PERIODIC SOLUTIONS OF THE PROBLEM OF THREE BODIES WITH APPLICATION TO THE LUNAR THEORY*
}

BY

\author{
FOREST RAY MOULTON.
}

1. Introduction.

2. The Differential Equations.

3. Existence of the Periodic Solutions.

4. Practical Construction of the Solutions.

5. Applications to the lunar Theory.

6. Applications to Darwin's Periodic Orbits.

\section{Introduction.}

This paper treats certain periodic solutions of the problem of three finite masses which move in the same plane under their mutual attractions. The forces are supposed to vary according to the Newtonian law, and it is assumed that the bodies are spheres. The periods of the solutions are equal to the synodical periods of the bodies, and the orbits reduce to circles when the dis turbing forces are put equal to zero. It follows that they are of the class called by PoIncare " Solutions de la première sorte." $\dagger$

In the series which have been developed for expressing the coördinates of the moon there are certain terms whose period is the same as that of its synodical revolution. In the notation of Delaunar $\ddagger$ they are the terms which involve $\sin k \mathrm{D}$ and $\cos k \mathrm{D}$, where $k$ is an integer. It is shown that ihese terms belong to the periodic orbits treated in this paper. By putting the mass of the moon equal to zero and transforming the parameter in terms of which the expansions are made, we can compare the coefficients of the various terms with those given by Delaunay.§

As applied to the Lunar Theory the results obtained in this paper are, when the mass of the moon is neglected, coextensive with those given by HILL in his Researches, $\|$ and by Brown in his first memoir. $\mid$

* Presented to the Society under a different title, September 8, 1905 and April 14, 1906. Received for publication June 26, 1906.

† Les Méthodes Nouvelles de la Mécanique Céleste, vol. 1, p. 97.

¥ Mémoires de l'A cadémie des Sciences, vol. 29 (1867), p. 801.

§ Loc. cit., pp. 803-924.

\| A merican Journal of Mathematics, vol. 1 (1878), pp. 5-26, 129-147, 245-260

TI A merican Journal of Mathematics, vol. 14 (1892), pp. 141-160. 
When the masses of two of the bodies have the ratio 1 to 10 , while that of the third is infinitesimal, the problem of this paper reduces to that which DARwin treated by numerical processes. ${ }^{*}$ For the sake of comparison, one of his "Satellite $A$ " orbits and one of his "Planet $A$ " orbits have been computed from the literal series derived below. A retrograde satellite orbit having the same sidereal period is also given. Darwin's "Satellites $B$ " and "Satellites $C$ " are imaginary for small values of the disturbing forces. They belong to values of the parameter which lie beyond that for which the series have a singularity, and their coördinates, therefore, are not represented by these series. There are only three classes of orbits of the type in question, real or imaginary, in which the bodies revolve in the forward direction, and it appears, therefore, that DARwin's search for these periodic satellite orbits was exhaustive. However, the number of retrograde orbits is the same as the number of those which revolve in the forward direction.

After having selected variables which seem most convenient for the construction of the series and for practical use, the existence of the solutions of the type in question is established. For this purpose the process of analytical continuation is employed which PoIncare developed in this general connection, and which he made the central idea of his researches on the figures of equilibrium of rotating fluid masses $\nmid$ and on the problem of three bodies. $\ddagger$ After the demonstrations of the existence and the properties of the solutions have been made, the practical construction of the series is carried out along lines which have been shown to be legitimate. Instead of arranging the solutions as Fourier series, as has been customary among astronomers, power series are employed, and the work has the simplicity which is characteristic of processes involving power series. For example, every coefficient is determined by a single step and is modified by no subsequent operations. Moreover, it is possible to prove the convergency of the series within certain limits, though the precise limits where convergency ceases have not been found.

\section{The Differential EQUations.}

Let the three masses be $E, M$, and $S$. Choosing $E$ as the origin and letting the rectangular coördinates of $M$ and $S$ be $x, y$ and $X, Y$ respectively, we have the differential equations of motion :

$$
\frac{d^{2} x}{d t^{2}}+k^{2}(E+M) \frac{x}{r^{3}}=-k^{2} S\left\{\frac{x-X}{\rho^{3}}+\frac{X}{R^{3}}\right\},
$$

* Acta Mathematica, vol. 21 (1897), pp. 99-242; Mathematische Annalen, vol. 51 (1898-9), pp. 523-583.

† Acta Mathematica, vol. 7 (1885), pp. 259-380.

$\ddagger$ Bulletin Astronomique, vol. 1 (1881). pp. 65-74; Acta Mathematica, vol. 13 (1890), pp. 8-270; Les Méthodes Nouvelles de la Mécanique Céleste (1892-9). 
where

$$
\begin{aligned}
& \frac{d^{2} y}{d t^{2}}+k^{2}(E+M) \frac{y}{r^{3}}=-k^{2} S\left\{\frac{y-Y}{\rho^{3}}+\begin{array}{c}
Y \\
R^{3}
\end{array}\right\}, \\
& \frac{d^{2} X}{d t^{2}}+k^{2}(E+S) \frac{X}{R^{3}}=-k^{2} M\left\{\frac{X-x}{\rho^{3}}+\frac{x}{r^{3}}\right\}, \\
& \frac{d^{2} Y}{d t^{2}}+k^{2}(E+S) \frac{Y}{R^{3}}=-k^{2} M\left\{\frac{Y-y}{\rho^{3}}+\frac{y}{r^{3}}\right\},
\end{aligned}
$$

$$
r=\sqrt{x^{2}+\overline{y^{2}}}, \quad \rho=\sqrt{(x-X)^{2}+(y-\bar{Y})^{2}}, \quad R=\sqrt{X^{2}+Y^{2}} .
$$

We shall now refer the motion of $S$ to the center of gravity of $E$ and $M$ by the transformation

$$
X=x^{\prime}+\frac{M}{E+M} x, \quad Y=y^{\prime}+\frac{M}{E+M} y, \quad r^{\prime}=\sqrt{x^{\prime 2}+y^{\prime 2}} .
$$

Then passing to polar coördinates by the transformation

$$
\begin{array}{ll}
x=r \cos v, & x^{\prime}=r^{\prime} \cos v^{\prime}, \\
y=r \sin v, & y^{\prime}=r^{\prime} \sin v^{\prime},
\end{array}
$$

the differential equations become

$$
\begin{aligned}
& \frac{d^{2} r}{d t^{2}}-r\left(\frac{d v}{d t}\right)^{2}+\frac{k^{2}(E+M)}{r^{2}}=k^{2} S(E+M) \frac{\partial u}{\partial r} \\
& r \frac{d^{2} v}{d t^{2}}+2 \frac{d r}{d t} \frac{d v}{d t}=k^{2} S(E+M) \frac{1}{r} \cdot \frac{\partial u}{\partial v} \\
& \frac{d^{2} r^{\prime}}{d t^{2}}-r^{\prime}\left(\frac{d v^{\prime}}{d t}\right)^{2}+\frac{k^{2} E(E+M+S)}{(E+M) r^{\prime 2}}=\frac{k^{2}(E+M+S)}{E+M} \frac{\partial u^{\prime}}{\partial r} \\
r^{\prime} & \frac{d^{2} v^{\prime}}{d t^{2}}+2 \frac{d r^{\prime}}{d t} \frac{d v^{\prime}}{d t}=\frac{k^{2}(E+M+S)}{E+M} \frac{1}{r^{\prime}} \frac{\partial u_{*}^{\prime}}{\partial v^{\prime}}
\end{aligned}
$$

where

$$
\begin{aligned}
& u=\frac{1}{E \rho}+\frac{1}{M R}, \\
& u^{\prime}=E\left(\frac{1}{\bar{R}}-\frac{1}{r^{\prime}}\right)+\frac{M}{\rho}, \\
& \rho=r^{\prime}\left[1-\frac{2 E}{E+M} \overline{r^{\prime}} \cos \left(v-v^{\prime}\right)+\left(\frac{E}{E+M}\right)^{2}\left(\frac{r}{r^{\prime}}\right)^{2}\right]^{t}, \\
& R=r^{\prime}\left[1+\frac{2 M}{E+M} \frac{r}{r^{\prime}} \cos \left(v-v^{\prime}\right)+\left(\frac{M}{E+M}\right)^{2}\left(\begin{array}{c}
r \\
r^{\prime}
\end{array}\right)^{2}\right]^{1} .
\end{aligned}
$$

Trans. Am. Math. Soc. 36 
If $r / r^{\prime}<1$ then $1 / R-1 / r^{\prime}$ and $1 / \rho$ can be expanded as converging power series in $r / r^{\prime}$ for all values of $v$ and $v^{\prime}$. Only cases in which this inequality is satisfied will be treated. In the Lunar Theory $r / r^{\prime}$ is about $\frac{1}{4} \frac{1}{0}$, and in the satellite orbits computed by DARwIN, about $\frac{1}{10}$.

The series for $u$ and $u^{\prime}$ are

$$
\begin{aligned}
& u=\frac{1}{r^{\prime}}\left\{\frac{1}{E}+\frac{1}{M}+\frac{E+M}{(E+M)^{2}}\left(\frac{r}{r^{\prime}}\right)^{2} \frac{1}{4}\left[1+3 \cos 2\left(v-v^{\prime}\right)\right]\right. \\
& +\frac{\left(E^{2}-M^{2}\right)}{(E+M)^{3}}\left(\frac{r}{r^{\prime}}\right)^{3} \frac{1}{8}\left[3 \cos \left(v-v^{\prime}\right)+5 \cos 3\left(v-v^{\prime}\right)\right] \\
& +\frac{E^{3}+M^{3}}{(E+M)^{4}}\left(\frac{r}{r^{\prime}}\right)^{4} \frac{1}{64}\left[9+20 \cos 2\left(v-v^{\prime}\right)+35 \cos 4\left(v-v^{\prime}\right)\right] \\
& +\frac{E^{4}-M^{4}}{(E+M)^{5}}\left(\frac{r}{r^{\prime}}\right)^{5} \frac{1}{12}\left[30 \cos \left(v-v^{\prime}\right)+35 \cos 3\left(v--v^{\prime}\right)+63 \cos 5\left(v-v^{\prime}\right)\right] \\
& +\cdots\} \\
& u^{\prime}=\frac{1}{r^{\prime}}\left\{M+\frac{E M}{4}\left(\frac{r}{r^{\prime}}\right)^{2}\left[1+3 \cos 2\left(v-v^{\prime}\right)\right]+\cdots\right\} .
\end{aligned}
$$

With these values of $u$ and $u^{\prime}$ equations (i) become

$$
\begin{aligned}
& \frac{d^{2} r}{d t^{2}}-r\left(\frac{d v}{d t}\right)^{2}+\frac{k^{2}(E+M)}{r^{2}}=\frac{k^{2} S}{2} \frac{r}{r^{\prime 3}}\left\{\left[1+3 \cos 2\left(v-v^{\prime}\right)\right]\right. \\
& \left.+\frac{3}{4} \frac{(E-M)}{(E+M)} \frac{r}{r^{\prime}}\left[3 \cos \left(v-v^{\prime}\right)+5 \cos 3\left(v-v^{\prime}\right)\right]+\cdots\right\}, \\
& r \frac{d^{2} v}{d t^{2}}+2 \frac{d r}{d t} \frac{d v}{d t}=-\frac{k^{2} S}{2} \frac{r}{r^{\prime 3}}\left\{3 \sin 2\left(v-v^{\prime}\right)\right. \\
& \left.+\frac{3}{4} \frac{(E-M)}{(E+M)} \frac{r}{r^{\prime}}\left[\sin \left(v-v^{\prime}\right)+5 \sin 3\left(v-v^{\prime}\right)\right]+\cdots\right\}, \\
& \begin{aligned}
\frac{d^{2} r^{\prime}}{d t^{2}}-r^{\prime}\left(\frac{d v^{\prime}}{d t}\right)^{2}+\frac{k^{2}(E+M+S)}{r^{\prime 2}} & =-\frac{k^{2} E M(E+M+S)}{2(E+M)^{2}} \\
& \times \frac{r^{2}}{r^{\prime 4}}\left\{\left[1+3 \cos 2\left(v-v^{\prime}\right)\right]+\cdots\right\},
\end{aligned} \\
& r^{\prime} \frac{d^{2} v^{\prime}}{d t^{2}}+2 \frac{d r^{\prime}}{d t} \frac{d v^{\prime}}{d t}=\frac{k^{2} E M(E+M+S)}{2(E+M)^{2}} \frac{\dot{r}^{2}}{r^{\prime 2}}\left\{3 \sin 2\left(v-v^{\prime}\right)+\cdots\right\}
\end{aligned}
$$


When the right members of these differential equations are neglected, that is, when $M$ is supposed to revolve around $E$ without having its motion disturbed by $S$ and when $S$ is supposed to revolve around the center of gravity of $E$ and $M$ without having its motion disturbed because of the distribution of the mass between the two separate bodies $E$ and $M$, a particular solution of the equations is

$$
\begin{gathered}
r=a, \quad v=\frac{k \sqrt{E+M} t=n t,}{a^{\ddagger}} t=v^{\prime}, \quad v^{\prime}=\frac{k \sqrt{E+M+S}}{a^{\prime 3}} t=n^{\prime} t,
\end{gathered}
$$

where $n$ and $n^{\prime}$ are the angular motions of $E$ and $S$ respectively. In developing the periodic solutions from the differential equations including the right members, the mean angular motions will be kept the same as in the undisturbed orbits, and $n$ and $n^{\prime}$ will be regarded as constants which are given by observation (in the applications to the Lunar Theory), or which are assumed. The masses are regarded as given, and the constants $a$ and $a^{\prime}$ are defined by the equations

$$
n^{2} a^{3}=k^{2}(E+M), \quad n^{\prime 2} a^{\prime 3}=k^{2}(E+M+S) .
$$

New variables $p, w, p^{\prime}$ and $w^{\prime}$ will be defined by the equations

$$
\begin{aligned}
r & =a(1+p), & v & =n t+w, \\
r^{\prime} & =a^{\prime}\left(1+p^{\prime}\right), & v^{\prime} & =n^{\prime} t+w^{\prime} .
\end{aligned}
$$

It follows that $a p, w, a^{\prime} p^{\prime}$ and $w^{\prime}$ are the deviations from the circular orbits of $M$ and $S$ due to their mutual perturbations. To reduce the differential equations to a still more convenient form we shall let

$$
\begin{aligned}
m & =\frac{n^{\prime}}{n-n^{\prime}}, \text { whence } n=\frac{n^{\prime}(1+m)}{m}, \\
\tau & =\left(n-n^{\prime}\right) t=\frac{n^{\prime}}{m} t, \\
\eta & =\frac{S}{E+M+S} .
\end{aligned}
$$

It follows from (4) and the definition of $m$ that

$$
\frac{a}{a^{\prime}}=\left(\frac{m}{1+m}\right)^{3}\left(\frac{E+M}{E+M+S}\right)^{3}
$$

That is, the ratio $a / a^{\prime}$ is expressible in terms of the parameter $m$ and might be eliminated from the equations. But in the Lunar Theory $a / a^{\prime}$ is regarded as 
a separate parameter and is retained explicitly. In order to keep the results in such a form that comparison may be made with the work of Delaunay, and at the same time to avoid the inconvenience of having power series in two parameters, we shall put in general

$$
\frac{E^{j}-(-1)^{j} M^{j}}{(E+M)^{j}}\left(\frac{a}{a^{\prime}}\right)^{j-1}=M_{j-1} m^{2(j-1)}, \quad\left(\frac{a}{a^{\prime}}\right)^{j}=N_{j} m^{2 j},
$$

where the $M_{j}$ and $N_{j}$ are determined so that (7) shall be fulfilled. The $m$ will be regarded as a variable parameter in the argument, and the $M_{j}$ and $N_{j}$ as fixed constants. Therefore the $m$ in (7) and the $m$ in (8) belong to different problems, except when they give the same value of $a / a^{\prime}$. At the end of the work this relation will always be fulfilled, and the equations (8) amount merely to an allowable generalization of the meaning of $m$. The relations have been selected so that the $M_{j}$ and $N_{j}$ are approximately $\left(\frac{3}{8}\right)^{j}$ in the case of the Lunar Theory, and the power of $m$ indicates the order of the term. With these substitutions the differential equations become

$$
\begin{aligned}
& \frac{d^{2} p}{d \tau^{2}}-(1+p)\left(1+m+\frac{d w}{d \tau}\right)^{2}+\frac{(1+m)^{2}}{(1+p)^{2}}=\frac{m^{2} \eta}{2} \frac{1+p}{\left(1+p^{\prime}\right)^{3}}\left\{\left[1+3 \cos 2\left(\tau+w-w^{\prime}\right)\right]\right. \\
& +\frac{3}{4} M_{1} m^{2} \frac{1+p}{1+p^{\prime}}\left[3 \cos \left(\tau+w-w^{\prime}\right)+5 \cos 3\left(\tau+w-w^{\prime}\right)\right] \\
& +\frac{1}{8} M_{2} m^{4} \frac{(1+p)^{2}}{\left(1+p^{\prime}\right)^{2}}\left[9+20 \cos 2\left(\tau+w-w^{\prime}\right)+35 \cos 4\left(\tau+w-w^{\prime}\right)\right] \\
& +\frac{5}{64} M_{3} m^{6} \frac{(1+p)^{3}}{\left(1+p^{\prime}\right)^{3}}\left[30 \cos \left(\tau+w-w^{\prime}\right)+35 \cos 3\left(\tau+w-w^{\prime}\right)\right. \\
& \left.\left.+1+63 \cos 5\left(\tau+w-w^{\prime}\right)\right]+\cdots\right\} \\
& +p) \frac{d^{2} w}{d \tau^{2}}+2 \frac{d p}{d \tau}\left(1+m+\frac{d w}{d \tau}\right)=-\frac{m^{2} \eta(1+p)}{2} \frac{\left(1+p^{\prime}\right)^{3}}{\left(1+\sin 2\left(\tau+w-w^{\prime}\right)\right.} \\
& +\frac{3}{4} M_{1} m^{2} \frac{1+p}{1+p^{\prime}}\left[\sin \left(\tau+w-w^{\prime}\right)+5 \sin 3\left(\tau+w-w^{\prime}\right)\right] \\
& +\frac{5}{8} M_{2} m^{4} \frac{(1+p)^{2}}{\left(1+p^{\prime}\right)^{2}}\left[2 \sin 2\left(\tau+w-w^{\prime}\right)+7 \sin 4\left(\tau+w-w^{\prime}\right)\right] \\
& +\frac{15}{64} M_{3} m^{6} \frac{(1+p)^{3}}{\left(1+p^{\prime}\right)^{3}}\left[2 \sin \left(\tau+w-w^{\prime}\right)+7 \sin 3\left(\tau+w-w^{\prime}\right)\right. \\
& \left.\left.\quad+21 \sin 5\left(\tau+w-w^{\prime}\right)\right]+\cdots\right\}
\end{aligned}
$$




$$
\begin{aligned}
& \frac{d^{2} p^{\prime}}{d \tau^{2}}-\left(1+p^{\prime}\right)\left(m+\frac{d w^{\prime}}{d \tau}\right)^{2}+\frac{m^{2}}{\left(1+p^{\prime}\right)^{2}}=-\frac{E M N_{2}}{2(E+M)^{2}} m^{6} \frac{(1+p)^{2}}{\left(1+p^{\prime}\right)^{4}} \\
& \times\left\{\left[1+3 \cos 2\left(\tau+w-w^{\prime}\right)\right]+\cdots\right\}, \\
& \left(1+p^{\prime}\right) \frac{d^{2} w^{\prime}}{d \tau^{2}}+2 \frac{d p^{\prime}}{d \tau}\left(m+\frac{d w^{\prime}}{d \tau}\right)=\frac{E M N_{2}}{2(E+M)^{2}} m^{6} \frac{(1+p)^{2}}{\left(1+p^{\prime}\right)^{4}} \\
& \times\left\{3 \sin 2\left(\tau+w-w^{\prime}\right)+\cdots\right\} .
\end{aligned}
$$

These equations are general for the determination of the motion of three mutually attracting spheres except for the condition that $a(1+p)$ shall be less than $a^{\prime}\left(1+p^{\prime}\right)$ throughout the motion.

\section{Existence of the periodic solutions.}

When $m=0$ equations (9) admit the periodic solution $p=w=p^{\prime}=w^{\prime}=0$. It will now be proved that for $m \neq 0$, but sufficiently small, the equations admit a periodic solution expansible as power series in $m$ and vanishing with $m$. It is the analytic continuation of the solution $p=w=p^{\prime}=w^{\prime}=m=0$ with respect to $m$ as the parameter. Since $\tau$ enters explicitly in (9) under the sine and cosine functions in integral multiples, it follows that the period of the solution must be a multiple of $2 \pi$.

It can easily be shown from the way in which $p, w, p^{\prime}, w^{\prime}$, and $\tau$ enter in (9) that if we impose the conditions $d p / d \tau=w=d p^{\prime} / d \tau=w=0$ at $\tau=j \pi$, then $p$ and $p^{\prime}$ are even functions of $\tau-j \pi$, and $w$ and $w^{\prime}$ are odd functions of $\tau-j \pi$. This means geometrically that at $\tau=j \pi$ the bodies are in a line and are crossing it perpendicularly, and that the orbits of $M$ and $S$ are respectively symmetrical with respect to axes coinciding with this line at $\tau=j \pi$ and rotating around $E$ and the center of gravity of $E$ and $M$ at any constant rate. Orbits of this type, whether they are periodic or not, will be called symmetrical orbits.

The existence of symmetrical periodic orbits will be established first. Suppose that, at $\tau=0$,

$$
\begin{aligned}
r=a(1+\nu) & =a(1+\alpha)(1-e), & r^{\prime}=a^{\prime}\left(1+p^{\prime}\right) & =a\left(1+\alpha^{\prime}\right)\left(1-e^{\prime}\right), \\
\overline{d r} & =a \frac{d p}{d \tau}=0, & \frac{d r^{\prime}}{d \tau} & =a^{\prime} \frac{d p^{\prime}}{d \tau}=0, \\
w & =0, & w^{\prime} & =0, \\
1+m+\frac{d w}{d \tau} & =\frac{(1+m) v \overline{1+e}}{(1+\alpha)^{\frac{3}{2}}(1-e)^{\frac{3}{2}}}, & m+\frac{d w^{\prime}}{d \tau} & =\frac{m \sqrt{1+e^{\prime}}}{\left(1+\alpha^{\prime}\right)^{\frac{3}{2}}}\left(1-e^{\prime}\right)^{\frac{3}{2}}
\end{aligned}
$$

If the right members of equations (9) are neglected, and if we put $r=a(1+p)$, $(1+m) \tau+w=u, r^{\prime}=a^{\prime}\left(1+p^{\prime}\right), m \tau+w^{\prime}=u^{\prime}$, then the left members reduce 
to two sets of equations for the two body problem. It is found that, with the initial conditions (10), $a \alpha$, and $a^{\prime} \alpha^{\prime}$ are the increments to the semi-axes of the orbits of $M$ and $S$ respectively, and that $e$ and $e$ are the eccentricities of their orbits. Because of the well-known properties of the solutions in terms of these parameters, the properties of the general solutions so far as they do not depend upon the right members of (9) can easily be written down. For this reason these particular parameters were selected.

Equations (9) are to be integrated as power series in $\alpha, e, \alpha^{\prime}, e^{\prime}$ and $m$, vanishing with these parameters. It follows from their form and PoINCARE's extension * of CAUCHY's theorem that, when an arbitrary interval for $\tau$ has been chosen in advance, these parameters can be assigned such small moduli that the series will converge uniformly as $\tau$ takes all values in the interval. We shall choose as the interval $0 \ldots 2 k \pi$ ( $k$ an integer) and the solution may be written

$$
\begin{aligned}
& p=\mathfrak{p}_{1}\left(\alpha, e, \alpha^{\prime}, e^{\prime}, m ; \tau\right), \\
& \frac{d p}{d \tau}=\mathfrak{P}_{2}\left(\alpha, e, \alpha^{\prime}, e^{\prime}, m ; \tau\right) \text {, } \\
& w=\mathfrak{p}_{3}\left(\alpha, e, \alpha^{\prime}, e^{\prime}, m ; \tau\right), \\
& \frac{d w}{d \tau}=\mathfrak{p}_{4}\left(\alpha, e, \alpha^{\prime}, e^{\prime}, m ; \tau\right), \\
& p^{\prime}=p_{5}\left(\alpha, e, \alpha^{\prime}, e^{\prime}, m ; \tau\right), \\
& \frac{d w^{\prime}}{d \tau}=\mathfrak{p}_{8}\left(\alpha, e, \alpha^{\prime}, e^{\prime}, m ; \tau\right),
\end{aligned}
$$

where $\mathfrak{p}_{1}, \cdots, \mathfrak{p}_{8}$ are power series in $\alpha, e, \alpha^{\prime}, e^{\prime}$, and $m$. The independent variable $\tau$ enters in the coefficients.

It follows from the symmetry of the orbits under the initial conditions (10) that if at $\tau=k \pi$ the three bodies are again in a line and are crossing the line perpendicularly, then the orbits necessarily reënter at $\tau=2 k \pi$, and conversely. Hence the necessary and sufficient conditions for a symmetrical periodic solution of period $2 k \pi$ are

$$
\mathfrak{p}_{i}\left(\alpha, e, \alpha^{\prime}, e^{\prime}, m ; k \pi\right)=0 \quad(i=2,3,6,7) .
$$

Since $\mathfrak{p}_{2}=\mathfrak{p}_{3}=\mathfrak{p}_{6}=\mathfrak{p}_{7}=0$ at $\tau=0$, it follows that equations (12) are identically satisfied by $\alpha=e=\alpha^{\prime}=e^{\prime}=m=0$. It will be shown that they can be solved for $\alpha, e, \alpha^{\prime}$ and $e^{\prime}$ as power series in $m$, vanishing with $m$, and converging for sufficiently small values of $m$. When the results of these solutions

\footnotetext{
*Acta Mathematioa, vol. 13 (1890), p. 16 ; Les Methodes Nouvelles de la Mécanique Céleste, vol. 1, p. 58.
} 
are substituted in (11), the coördinates will be expressed as power series in $m$ alone, and they will converge for sufficiently small values of $m$. The conditions for periodicity will have been satisfied, and therefore for $m$ sufficiently small, but not zero, the series will converge uniformly for all values of $\tau$. Since the series converge for a continuous range of values of $m$, the coefficient of each power will be periodic.

The first two equations of (9) do not carry $p^{\prime}$ and $w^{\prime}$ except in their right members; therefore $\mathfrak{p}_{1}, \ldots, \mathfrak{p}_{4}$ do not contain $\alpha^{\prime}$ and $e^{\prime}$ except in terms which are multiplied by $\mathrm{m}^{2}$. Likewise $\mathfrak{p}_{5}, \ldots, \mathfrak{p}_{8}$ do not involve $\alpha$ and e except in terms which are multiplied by $m^{6}$. All the terms in $\mathfrak{p}_{1}, \cdots, \mathfrak{p}_{4}$ which do not contain $m^{2}$ as a factor can be obtained from the left members of the first two equations of (9) set equal to zero. All the terms in $\mathfrak{p}_{5}, \ldots, \mathfrak{p}_{8}$ which do not contain $m^{6}$ as a factor can be obtained from the left members of the third and fourth equations of (9) set equal to zero. The left members of (9) set equal to zero constitute two pairs of equations of the two body problem, and making use of this fact and the initial conditions (10), we may write the solution (11):

$$
\begin{aligned}
p & =\mathfrak{p}_{1}=-e\left\{\cos \nu \tau+\frac{e}{2}(\cos 2 \nu \tau-1)+\cdots\right\}+m^{2} \mathfrak{p}_{1}^{\prime}\left(\alpha, e, \alpha^{\prime}, e^{\prime}, m ; \tau\right) ; \\
\frac{d p}{d \tau} & =\mathfrak{p}_{2}=e \nu\{\sin \nu \tau+e \sin 2 \nu \tau+\cdots\}+m^{2} \mathfrak{p}_{2}^{\prime}\left(\alpha, e, \alpha^{\prime}, e^{\prime}, m ; \tau\right), \\
w & =\mathfrak{p}_{3}=\{-(1+m) \tau+\nu \tau+2 e \sin \nu \tau+\cdots\}+m^{2} \mathfrak{p}_{3}^{\prime}\left(\alpha, e, \alpha^{\prime}, e^{\prime}, m ; \tau\right) \\
\frac{d w}{d \tau} & =\mathfrak{p}_{4}=\{-(1+m)+\nu+2 e \nu \cos \nu \tau+\cdots\}+m^{2} \mathfrak{p}_{4}^{\prime}\left(\alpha, e, \alpha^{\prime}, e^{\prime}, m ; \tau\right), \\
p^{\prime} & =\mathfrak{p}_{5}=-e^{\prime}\left\{\cos \nu^{\prime} \tau+\frac{e^{\prime}}{2}\left(\cos 2 \nu^{\prime} \tau-1\right)+\cdots\right\}+m^{6} \mathfrak{p}_{5}^{\prime}\left(\alpha, e, \alpha^{\prime}, e^{\prime}, m ; \tau\right), \\
\frac{d p^{\prime}}{d \tau} & =\mathfrak{p}_{6}=e^{\prime} \nu^{\prime}\left\{\sin \nu^{\prime} \tau+e^{\prime} \sin 2 \nu^{\prime} \tau+\cdots\right\}+m^{6} \mathfrak{p}_{6}^{\prime}\left(\alpha, e, \alpha^{\prime}, e^{\prime}, m ; \tau\right), \\
w^{\prime} & =\mathfrak{p}_{7}=\left\{-m \tau+\nu^{\prime} \tau+2 e^{\prime} \sin \nu^{\prime} \tau+\cdots\right\}+m^{6} \mathfrak{p}_{7}^{\prime}\left(\alpha, e, \alpha^{\prime}, e^{\prime}, m ; \tau\right), \\
\frac{d w^{\prime}}{d \tau} & =\mathfrak{p}_{8}=\left\{-m+\nu^{\prime}+2 e^{\prime} \nu^{\prime} \cos \nu^{\prime} \tau+\cdots\right\}+m^{6} \mathfrak{p}_{8}^{\prime}\left(\alpha, e, \alpha^{\prime}, e^{\prime}, m ; \tau\right),
\end{aligned}
$$

where

$$
\nu=\frac{1+m}{(1+\alpha)^{\prime}}, \quad \nu^{\prime}=\frac{m}{\left(1+\alpha^{\prime}\right)^{\prime}}
$$

The terms contained in $\mathfrak{p}_{1}^{\prime}, \ldots, \mathfrak{p}_{8}^{\prime}$ depend upon the right members of the differential equations. 
The conditions for periodic solutions (12) become

$$
\begin{aligned}
\mathfrak{p}_{2}(k \pi)=e v\{\sin \nu k \pi+e \sin 2 \nu k \pi+\cdots\} & +m^{2} \mathfrak{p}_{2}^{\prime}\left(\alpha, e, \alpha^{\prime}, e^{\prime}, m ; k \pi\right)=0, \\
\mathfrak{F}_{3}(k \pi)=\{-(1+m) k \pi+\nu k \pi+2 e \sin \nu k \pi+\cdots\} & +m^{2} \mathfrak{p}_{3}^{\prime}\left(\alpha, e, \alpha^{\prime}, e^{\prime}, m ; k \pi\right)=0, \\
\mathfrak{p}_{6}(k \pi)=e^{\prime} \nu\left\{\sin \nu^{\prime} k \pi+e^{\prime} \sin 2 \nu^{\prime} k \pi+\cdots\right\} & +m^{6} \mathfrak{p}_{6}^{\prime}\left(\alpha, e, \alpha^{\prime}, e^{\prime}, m ; k \pi\right)=0, \\
\mathfrak{p}_{7}(k \pi)=\left\{-m k \pi+\nu^{\prime} k \pi+2 e^{\prime} \sin \nu^{\prime} k \pi\right. & +\cdots\} \\
& +m^{6} \mathfrak{p}_{7}^{\prime}\left(\alpha, e, \alpha^{\prime}, e^{\prime}, m ; k \pi\right)=0 .
\end{aligned}
$$

All the unwritten terms in the \{\} involve sines of multiples of $\nu k \pi$ or $\nu^{\prime} k \pi$; those in the first two \{\} carry $e^{2}$ as a factor, and in the last two, $e^{\prime 2}$.

Substituting the expression for $\nu^{\prime}$ as defined in (14) in the last two equations of $(15)$, we find that $\mathfrak{p}_{6}(k \pi)$ is divisible by $m^{2}$ and that $\mathfrak{p}_{7}(k \pi)$ is divisible by $m$. After division by these factors there is no term independent of $\alpha^{\prime}, e^{\prime}$, and $m$, and the determinant of the linear terms in $\alpha^{\prime}$ and $e^{\prime}$ is

$$
\Delta \equiv\left|\begin{array}{cc}
0, & k \pi \\
-{ }_{2}^{3} k \pi, & 2 k \pi
\end{array}\right|
$$

which is distinct from zero. An exception can occur only if $m k$ is an integer, when the determinant does vanish. But since $k$ may be put equal to unity this requires that $m$ shall be an integer, a case which arises for no satellite and for which the series derived are almost certainly always divergent. Hence it will be supposed that $m$ is not an integer. Since $\Delta$ is not zero, $\mathfrak{p}_{6}(k \pi) / m^{2}=0$ and $\mathfrak{p}_{7}(k \pi) / m^{2}=0$ may be solved uniquely for $\alpha^{\prime}$ and $e^{\prime}$ as power series in $\alpha, e$, and $m$. These equations are satisfied by $\alpha^{\prime}=e^{\prime}=m=0$ whatever $\alpha$ and $e$ may be, and therefore the solutions contain $m$ as a factor, and may be written

$$
\alpha^{\prime}=m q_{1}(\alpha, e, m), \quad e^{\prime}=m q_{2}(\alpha, e, m) \text {. }
$$

When these expressions for $\alpha^{\prime}$ and $e^{\prime}$ are substituted in the first two equations of (15) the latter become

$$
\begin{aligned}
& \mathfrak{p}_{2}(k \pi)=e \nu\{\sin \nu k \pi+e \sin 2 \nu k \pi+\cdots\}+m^{2} \mathfrak{p}_{2}^{\prime \prime}(\alpha, e, m ; k \pi)=0, \\
& \mathfrak{p}_{3}(k \pi)=\{-(1+m) k \pi+\nu k \pi+2 e \sin \nu k \pi+\cdots\}+m^{2} \mathfrak{p}_{3}^{\prime \prime}(\alpha, e, m ; k \pi)=0 .
\end{aligned}
$$

Before solving equations (16) we shall find the degree of the lowest terms in $m$ alone which they contain. The terms which are independent of $\alpha, e, \alpha^{\prime}$ and $e^{\prime}$ are found by integrating equations (9) in series of the form

$$
p=\sum_{i=1}^{\infty} p_{i} m^{i}, \quad w=\sum_{i=1}^{\infty} w_{i} m^{i}, \quad p^{\prime}=\sum_{i=1}^{\infty} p_{i}^{\prime} m^{i}, \quad w^{\prime}=\sum_{i=1}^{\infty} w_{i}^{\prime} m^{i}
$$


Substituting these expressions in (9), equating coefficients of corresponding powers of $m$, integrating the resulting equations, and imposing the initial conditions (10), we find

$$
\begin{aligned}
& p_{1}=w_{1}=0, \\
& p_{2}=-\eta+2 \eta \cos \tau-\eta \cos 2 \tau, \\
& w_{2}=\frac{5}{4} \eta \tau-4 \eta \sin \tau+\frac{11}{8} \eta \sin 2 \tau, \\
& p_{3}=\frac{1}{2} \eta+\frac{2}{3} \eta \cos \tau-\frac{7}{6} \eta \cos 2 \tau-2 \eta \tau \sin \tau, \\
& w_{3}=\eta \tau-\frac{4}{3} \eta \sin \tau+\frac{13}{6} \eta \sin 2 \tau-4 \eta \tau \cos \tau .
\end{aligned}
$$

These expressions give, at $\tau=k \pi$,

$$
\begin{array}{ll}
\frac{d p_{1}}{d \tau}=w_{1}=\frac{d p_{2}}{d \tau}=0, & w_{2}=\frac{5}{4} \eta k \pi, \\
\frac{d p_{3}}{d \tau}=-(-1)^{k} 2 \eta k \pi, & w_{3}=\eta k \pi-(-1)^{k} 4 \eta k \pi .
\end{array}
$$

Therefore the lowest power of $m$ contained in the first equation of (16) is the third, and in the second equation, the second.

Substitute in (16) the expression for $\nu$ given in (14) and consider the solution of the second equation. Every term contains either $m$ or $\alpha$ as a factor. The lowest degree in $\alpha$ alone is the first, and in $m$ alone, the second. Therefore the equation can be solved for $\alpha$ as a power series in $m$ and $e$, containing $m^{2}$ as a factor. Thus

$$
\alpha=m^{2} q_{3}(e, m) .
$$

Let (17) be substituted in the first equation of (16). After the substitution it is found that a single factor $m$ can be divided out leaving a linear term in $e$ and a second degree term in $m$ as the lowest. Consequently the equation can be solved for $e$ as a power series in $m$, containing $m^{2}$ as a factor. Therefore we have

$$
e=m^{2} q_{4}(m) .
$$

By substituting back through the several steps of the solution, we obtain

$$
\begin{aligned}
\alpha & =m^{2} q_{1}^{\prime}(m), & e & =m^{2} q_{2}^{\prime}(m), \\
\alpha^{\prime} & =m^{2} q_{3}^{\prime}(m), & e^{\prime} & =m^{2} q_{4}^{\prime}(m),
\end{aligned}
$$

where $q_{1}^{\prime}, \ldots, q_{4}^{\prime}$ are power series in $m$. The first two contain terms independent of $m$; the question for the last two will be left open until the practical construction of the periodic series is considered, though it might be answered at this place. 
When the expressions (19) are substituted in equations (13) the periodic solution is obtained. The series for $p$ and $w$ start with terms of the second degree in $m$. When $k$ has been chosen the whole process is unique. Therefore for a given value of $m$ there is but one direct orbit of the type under consideration which reënters after $k$ synodic revolutions. This holds when $k=1$. But all the reëntrant orbits after $k$ revolutions include those which reënter after one revolution; therefore, since the result is unique for every $k$, all of the orbits of the type treated rë̈nter after a single synodic revolution.

If the orbit of $M$ is retrograde, $n$ is negative and $m$ has the definition

$$
m=\frac{-n^{\prime}}{n+n^{\prime}}
$$

With this definition of $m$ the discussion of the existence of symmetrical periodic orbits proceeds precisely as before, and it is found that they exist. Consequently, there is but one direct and but one retrograde orbit of the type in question with a given sidereal period. For the. retrograde orbit the modulus of $m$ is smaller than for the direct, and the deviations from a circular orbit are in general less.

Suppose the condition is no longer imposed that the orbits shall be symmetrical, and consider the question of the existence of periodic solutions. We may suppose that at $\tau=0$

$$
\begin{array}{llll}
p=\alpha, & w=0, & \frac{d p}{d \tau}=\beta, & \frac{d w}{d \tau}=\gamma, \\
p^{\prime}=\alpha^{\prime}, & w^{\prime}=0, & \frac{d p^{\prime}}{d \tau}=\beta^{\prime}, & \frac{d w^{\prime}}{d \tau}=\gamma^{\prime}
\end{array}
$$

That is, at the origin of time $E, M$ and $S$ are in a line, but the initial conditions are not otherwise defined. The conditions $w=w^{\prime}=0$ are no limitations upon the generality of the problem for they amount simply to a particular choice of the origin of time. The solutions may be expressed as power series in $\alpha, \beta, \gamma, \alpha^{\prime}, \beta^{\prime}, \gamma^{\prime}$ and $m$ whose moduli may be taken so small that the convergence holds for $\tau$ in the whole interval $0 \ldots 2 \pi$. The conditions for periodic solutions with period $2 \pi$ are

$$
\begin{aligned}
p\left(\alpha, \beta, \gamma, \alpha^{\prime}, \beta^{\prime}, \gamma^{\prime} ; 2 \pi\right) & =p\left(\alpha, \beta, \gamma, \alpha^{\prime}, \beta^{\prime}, \gamma^{\prime} ; 0\right)=\alpha, \\
\frac{d p}{d \tau}\left(\alpha, \beta, \gamma, \alpha^{\prime}, \beta^{\prime}, \gamma^{\prime} ; 2 \pi\right) & =\frac{d p}{d \tau}\left(\alpha, \beta, \gamma, \alpha^{\prime}, \beta^{\prime}, \gamma^{\prime} ; 0\right)=\beta, \\
\frac{d w^{\prime}}{d \tau}\left(\alpha, \beta, \gamma, \alpha^{\prime}, \beta^{\prime}, \gamma^{\prime} ; 2 \pi\right) & =\frac{d w^{\prime}}{d \tau}\left(\alpha, \beta, \gamma, \alpha^{\prime}, \beta^{\prime}, \gamma^{\prime} ; 0\right)=\gamma^{\prime} .
\end{aligned}
$$


Equations (9) admit two uniform integrals, that of vis viva and that of areas. They may be written

$$
\begin{aligned}
& F_{1}\left(p, \frac{d p}{d \tau}, w, \frac{d w}{d \tau}, p^{\prime}, \frac{d p^{\prime}}{d \tau}, w^{\prime}, \frac{d w^{\prime}}{d \tau}\right)=F_{1}\left(\alpha, \beta, \gamma, \alpha^{\prime}, \beta^{\prime}, \gamma^{\prime}, m\right), \\
& F_{2}\left(p, \frac{d p}{d \tau}, w, \frac{d w}{d \tau}, p^{\prime}, \frac{d p^{\prime}}{d \tau}, w^{\prime}, \frac{d w^{\prime}}{d \tau}\right)=F_{2}\left(\alpha, \beta, \gamma, \alpha^{\prime}, \beta^{\prime}, \gamma^{\prime}, m\right) .
\end{aligned}
$$

It follows from these relations that when six of equations (20) are satisfied the remaining two are reduced to identities.* Consequently two of the equations may be suppressed and the remaining six solved for $\alpha, \beta, \gamma, \alpha^{\prime}, \beta^{\prime}, \gamma^{\prime}$ in terms of $m$. These solutions furnish the periodic orbits.

In order to determine which equations may be most conveniently suppressed because of the existence of the integrals $F_{1}$ and $F_{2}$ some details are necessary. Likewise in solving the remaining six equations more suitable parameters must be employed. However, the details will all be omitted. It is known that the equations admit one solution for they include as a special case the symmetrical orbits. The detailed discussion shows that they admit but one solution. Therefore the periodic orbits of the type in question are all symmetrical.

\section{Practical construction of the periodic solutions.}

The demonstrations of the existence of the periodic solutions carry with them possible, but very laborious, methods of constructing the series. A direct and convenient method of computing the coefficients will now be developed.

It has been proved that the periodic solutions are expressible in the form

$$
\begin{aligned}
p & =\sum_{i=2}^{\infty} p_{i} m^{i}, & w & =\sum_{i=2}^{\infty} w_{i} n \iota^{i}, \\
p^{\prime} & =\sum_{i=2}^{\infty} p_{i}^{\prime} m^{\prime}, & w^{\prime} & =\sum_{i=2}^{\infty} w_{i}^{\prime} m^{i} .
\end{aligned}
$$

The derivatives are expressible in the same form, and all the series converge for all values of $m$ whose moduli are sufficiently small. It has been shown that the coefficients of (21) can be uniquely determined so that they shall fulfil the three following conditions :

(a) The series satisfy the differential equation (9); and, because these conditions are fulfilled uniformly over a finite interval in $m$, they satisfy the differential equations identically in $m$.

(b) The series are periodic ; and, because the periodicity holds for a continuous range of values of $m$, each $p_{i}, w_{i}, p_{i}^{\prime}, w_{i}^{\prime}$ is separately periodic.

* Compare Les Méthodes Nouvelles de la Mécanique Céleste, vol. 1, p. 88. 
(c) Since the orbits are symmetrical the origin of time may be chosen so that $d p / d \tau=w=d p^{\prime} / d \tau=w^{\prime}=0$ at $\tau=0$. Since this is true for all values of $m$ whose moduli are sufficiently small, ea $\cap h d p_{i} / d \tau, w_{i}, d p_{i}^{\prime} / d \tau$ and $w_{i}^{\prime}$ separately vanishes at $\tau=0$.

These conditions will be referred to simply as $(a),(b)$ and $(c)$.

Equations (21) are to be substituted in (9) and the results rearranged as power series in $m$. It is most convenient to make these preliminary expansions as far as they will be required before the integration is taken up. In the induction which follows we shall need the terms in $p$ and $w$ to the fourth power, and in $p^{\prime}$ and $w^{\prime}$ to the sixth. Up to this order we have from (9) and (21)

$$
\begin{aligned}
& +(1+p)\left(1+m+\frac{d w}{d \tau}\right)^{2}=[1]+[2] m+\left[1+2 \frac{d w_{2}}{d \tau}+p_{2}\right] m^{2} \\
& +\left[2\left(\frac{d w_{3}}{d \tau}+\frac{d w_{2}}{d \tau}+p_{2}\right)+p_{3}\right] m^{3} \\
& +\left[2\left(\frac{\dot{d} w_{4}}{d \tau}+\frac{d w_{3}}{d \tau}+p_{2} \frac{d w_{2}}{d \tau}+p_{3}\right)+\left(\frac{d w_{2}}{d \tau}\right)^{2}+p_{2}+p_{4}\right] m^{4}+\cdots, \\
& -\frac{(1+m)^{2}}{(1+p)^{2}}=[-1]+[-2] m+\left[-1+2 p_{2}\right] m^{2}+\left[2 p_{3}+4 p_{2}\right] m^{3} \\
& +\left[2 p_{1}-3 p_{2}^{2}+4 p_{3}+2 p_{2}\right] m^{4}+\cdots, \\
& +\frac{m^{2} \eta}{2} \frac{(1+p)}{\left(1+p^{\prime}\right)^{3}}\left[1+3 \cos 2\left(\tau+w-w^{\prime}\right)\right]=\frac{m^{2} \eta}{2}\{[1+3 \cos 2 \tau] \\
& \left.+\left[p_{2}(1+3 \cos 2 \tau)-6 w_{2} \sin 2 \tau\right] m^{2}+\cdots\right\},
\end{aligned}
$$

$$
\begin{aligned}
& +\frac{3}{8} M_{1} m^{4} \eta \frac{(1+p)^{2}}{\left(1+p^{\prime}\right)^{4}}\left[3 \cos \left(\tau+w-w^{\prime}\right)+5 \cos 3\left(\tau+w-w^{\prime}\right)\right] \\
& =\frac{3}{8} M_{1} m^{4} \eta\{[3 \cos \tau+5 \cos 3 \tau]+\cdots\} \text {, } \\
& -(1+p) \frac{d^{2} w}{d \tau^{2}}=-\left[\frac{d^{2} w_{2}}{d \tau^{2}}\right] m^{2}-\left[\frac{d^{2} w_{3}}{d \tau^{2}}\right] m^{3}-\left[\frac{d^{2} w_{4}}{d \tau^{2}+p_{2}^{--} d \tau^{2}}\right] m^{4}+\cdots, \\
& -2 \frac{d p}{d \tau}\left(1+m+\frac{d w}{d \tau}\right)=-\left[2 \frac{d p_{2}}{d \tau}\right] m^{2}-\left[2 \frac{d p_{3}}{d \tau}+2 \frac{d p_{2}}{d \tau}\right] m^{3} \\
& -\left[2 \frac{d p_{4}}{d \tau}+2 \frac{d p_{3}}{d \tau}+2 \frac{d p_{2}}{d \tau} \frac{d w_{2}}{d \tau}\right] m^{4}+\cdots \\
& -\frac{3}{2} m^{2} \eta \frac{(1+p)}{\left(1+p^{\prime}\right)^{3}} \sin 2\left(\tau+w-w^{\prime}\right)=-\frac{3}{2} m^{2} \eta\{[\sin 2 \tau] \\
& \left.+\left[p_{2} \sin 2 \tau+2 w_{2} \cos 2 \tau\right] m^{2}+\cdots\right\} \\
& -\frac{3}{8} M_{1} m^{4} \eta \frac{(1+p)^{2}}{\left(1+p^{\prime}\right)^{4}}\left[\sin \left(\tau+w-w^{\prime}\right)+5 \sin 3\left(\tau+w-w^{\prime}\right)\right] \\
& =-\frac{3}{8} M_{1} \eta m^{4}\{[\sin \tau+5 \sin 3 \tau]+\cdots\} \text {, }
\end{aligned}
$$


and corresponding equations from the last two of (9) which need not be written down. It is a simple matter to write out the expansions as far as is desired. In the computations given in this paper the series were carried out so as to include all terms in $m^{10}$. It will be shown that after these series are written out there is no need of further reference to the differential equations.

It has been proved that there are no terms of the first degree in $m$. This result will be accepted though it would have come out in the construction if it were not already known. From (22) and condition $(a)$ it follows that the equations which define the coefficients of $m^{2}$ in the solution are

$$
\begin{array}{ll}
\frac{d^{2} p_{2}}{d \tau^{2}}-3 p_{2}-2 \frac{d w_{2}}{d \tau}=\frac{1}{2} \eta(1+3 \cos 2 \tau), & \frac{d^{2} p_{2}^{\prime}}{d \tau^{2}}=0, \\
\frac{d^{2} w_{2}}{d \tau^{2}}+2 \frac{d p_{2}}{d \tau}=-\frac{3}{2} \eta \sin 2 \tau, & \frac{d^{2} w_{2}^{\prime}}{d \tau^{2}}=0 .
\end{array}
$$

The general solutions of these equations are

$$
\begin{aligned}
& p_{2}=\frac{1}{2} \eta+2 c_{1}^{(2)}+c_{2}^{(2)} \cos \tau+c_{3}^{(2)} \sin \tau-\eta \cos 2 \tau, \\
& w_{2}=c_{4}^{(2)}-\left(3 c_{1}^{(2)}+\eta\right) \tau-2 c_{2}^{(2)} \sin \tau+2 c_{3}^{(2)} \cos \tau+\frac{11}{8} \eta \sin 2 \tau, \\
& p_{2}^{\prime}=c_{1}^{\prime(2)} \tau+c_{2}^{\prime(2)}, \\
& w_{2}^{\prime}=c_{3}^{\prime(2)} \tau+c_{4}^{\prime(2)},
\end{aligned}
$$

where $c_{1}^{(2)}, c_{2}^{(2)}, c_{3}^{(2)}, c_{4}^{(2)}, c_{1}^{\prime(2)}, c_{2}^{\prime(2)}, c_{3}^{(2)}$ and $c_{4}^{\prime(2)}$ are the constants of integration which are so far arbitrary.

It follows from condition $(b)$ that

$$
3 c_{1}^{(2)}+\eta=0, \quad c_{1}^{\prime(2)}=c_{3}^{\prime(2)}=0 ;
$$

and from condition $(c)$ that

$$
c_{3}^{(2)}=c_{4}^{(2)}=c_{4}^{(2)}=0 .
$$

Therefore equations (23) become

$$
\begin{array}{ll}
p_{2}=-\frac{1}{6} \eta+c_{2}^{(2)} \cos \tau-\eta \cos 2 \tau, & p_{2}^{\prime}=c_{2}^{\prime(2)}, \\
w_{2}=-2 c_{2}^{(2)} \sin \tau+\frac{11}{8} \eta \sin 2 \tau, & w_{2}^{\prime}=0,
\end{array}
$$

where $c_{2}^{(2)}$ and $c_{2}^{\prime(2)}$ are so far arbitrary.

The equations which determine the coefficients of $m^{3}$ are found from (22) to be

$$
\frac{d^{2} p_{3}}{d \tau^{2}}-3 p_{3}-2 \frac{d w_{3}}{d \tau}=2 \frac{d w_{2}}{d \tau}+6 p_{2}=-\eta+2 c_{2}^{(2)} \cos \tau-\frac{1}{2} \eta \cos 2 \tau,
$$




$$
\begin{aligned}
& \frac{d^{2} w_{3}}{d \tau^{2}}+2 \frac{d p_{3}}{d \tau}=-2 \frac{d p_{2}}{d \tau}=2 c_{2}^{(2)} \sin \tau-4 \eta \sin 2 \tau, \\
& \frac{d^{2} p_{3}^{\prime}}{d \tau^{2}}=0 \\
& \frac{d^{2} w_{3}^{\prime}}{d \tau^{2}}=0 .
\end{aligned}
$$

The general solutions of these equations are

$p_{3}=2 c_{1}^{(3)}-\eta+c_{2}^{(3)} \cos \tau+c_{3}^{(3)} \sin \tau-\frac{7}{6} \eta \cos 2 \tau-c_{2}^{(2)} \tau \sin \tau$,

$w_{3}=c_{4}^{(3)}+\left(-3 c_{1}^{(3)}+2 \eta\right) \tau-2 c_{2}^{(3)} \sin \tau+2 c_{3}^{(3)} \cos \tau+{ }_{6}^{1} \eta \sin 2 \tau-2 c_{2}^{(2)} \tau \cos \tau$, $p_{3}^{\prime}=c_{1}^{(3)} \tau+c_{2}^{\prime(3)}$,

$w_{3}^{\prime}=c_{3}^{\prime(3)} \tau+c_{4}^{\prime(3)}$,

where $c_{1}^{(3)}, \cdots, c_{4}^{\prime(3)}$ are so far arbitrary.

From conditions $(b)$ and $(c)$ we have

$$
\begin{aligned}
& c_{2}^{(2)}=c_{3}^{(3)}=c_{4}^{(3)}=c_{1}^{(3)}=c_{3}^{(3)}=c_{4}^{(3)}=0, \\
& -3 c_{1}^{(3)}+2 \eta=0,
\end{aligned}
$$

while $c_{2}^{(3)}$ and $c_{2}^{(3)}$ so far arbitrary.

Hence we have

$$
\begin{array}{ll}
p_{2}=-\frac{1}{6} \eta-\eta \cos 2 \tau, & p_{2}^{\prime}=c_{2}^{\prime(2)}, \\
w_{2}=\frac{1}{8} \eta \sin 2 \tau, & w_{2}^{\prime}=0, \\
p_{3}=\frac{1}{3} \eta+c_{2}^{(3)} \cos \tau-\frac{7}{6} \eta \cos 2 \tau, . & p_{3}^{\prime}=c_{2}^{\prime(3)}, \\
w_{3}=-2 c_{2}^{(3)} \sin \tau+{ }_{6}^{8} \eta \sin 2 \tau, & w_{3}^{\prime}=0 ;
\end{array}
$$

$p_{2}$ and $w_{2}$ are now completely determined.

It is necessary to go one step further in order to make the induction to the general formulas. The equations which determine the terms of the fourth order are, from (22),

$$
\begin{aligned}
\frac{d^{2} p_{4}}{d \tau^{2}}-3 p_{1} & -2 \frac{d w_{4}}{d \tau}=2 \frac{d w_{3}}{d \tau}+2 p_{2} \frac{d w_{2}}{d \tau}-3 p_{2}^{2}+6 p_{3}+\left(\frac{d w_{2}}{d \tau}\right)^{2}+3 p_{2} \\
& +\frac{1}{2} \eta p_{2}+\frac{3}{2} \eta p_{2} \cos 2 \tau-3 \eta w_{2} \sin 2 \tau+\frac{9}{8} M_{1} \eta \cos \tau+\frac{1}{8} M_{1} \eta \cos 3 \tau \\
= & {\left[-\frac{38}{96}-\eta^{2}+\frac{8}{2} \eta\right]+\left[2 c_{2}^{(3)}+\frac{9}{8} M_{1} \eta\right] \cos \tau-\frac{4}{8} \eta[1+2 \eta] \cos 2 \tau } \\
& +\frac{15}{8} M_{1} \eta \cos 3 \tau+\frac{2}{8} \frac{7}{2} \eta^{2} \cos 4 \tau,
\end{aligned}
$$


(26)

$$
\begin{aligned}
& \frac{d^{2} w_{4}}{d \tau^{2}}+2 \frac{d p_{4}}{d \tau}=-p_{2} \frac{d^{2} w_{2}}{d \tau^{2}}-2 \frac{d p_{3}}{d \tau}-2 \frac{d p_{2}}{d \tau} \frac{d w_{2}}{d \tau}-\frac{3}{2} \eta p_{2} \sin 2 \tau \\
& -3 \eta w_{2} \cos 2 \tau-\frac{8}{8} M_{1} \eta \sin \tau-\frac{15}{8} M_{1} \eta \sin 3 \tau \\
& =\left[2 c_{2}^{(3)}-\frac{3}{8} M_{1} \eta\right] \sin \tau-\frac{2}{3} \eta(7+\eta) \sin 2 \tau-\frac{15}{8} M_{1} \eta \sin 3 \tau-\frac{15}{16} \eta^{2} \sin 4 \tau, \\
& \frac{d^{2} p_{4}^{\prime}}{d \tau^{2}}=3 p_{2}^{\prime}=3 c_{2}^{\prime(2)} \\
& \frac{d^{2} w_{4}^{\prime}}{d \tau^{2}}=0
\end{aligned}
$$

Integrating the second equation once and substituting the expression for $d w_{4} / d \tau$ in the first, it is found that

$$
\begin{aligned}
\frac{d^{2} p_{4}}{d \tau^{2}}+p_{4}=\left[2 c_{1}^{(4)}-\frac{83}{96}-\eta^{2}+\frac{8}{2} \eta\right]+\left[-2 c_{2}^{(3)}+\frac{15}{8} M_{1} \eta\right] \cos \tau \\
+\frac{2}{3} \eta(5-3 \eta) \cos 2 \tau+\frac{25}{8} M_{1} \eta \cos 3 \tau+\frac{45}{8} \eta^{2} \cos 4 \tau
\end{aligned}
$$

In order that the solution of this equation may be periodic [condition $(b)$ ] the coefficient of $\cos \tau$ must be put equal to zero, or

$$
c_{2}^{(3)}=\frac{15}{16} M_{1} \eta
$$

Thus the undetermined coefficient which remained in $p_{3}$ and $w_{y}$ is uniquely determined by the condition that $p_{4}$ shall be periodic.

After $c_{2}^{(3)}$ has been determined by (28) the solution of (27) is periodic. When the condition has been imposed that $d p_{4} / d \tau$ shall equal zero at $\tau=0$, the expression for $p_{4}$ will carry, in addition to $c_{1}^{(t)}$, an undetermined coefficient $c_{2}^{(t)}$ with $\cos \tau$. When this value of $p_{4}$ is substituted in the integral of the second equation of (26) it takes the form

$$
\frac{d w_{4}}{d \tau}=\left[-3 c_{1}^{(4)}+\frac{83}{4} \frac{1}{8} \eta^{2}-3 \eta\right]+[] \cos \tau+\cdots
$$

In order that $w_{4}$ may be periodic the constant part of the right member of this equation must vanish, which uniquely determines $c_{1}^{(4)}$. Then $p_{4}$ and $w_{4}$ carry a single undetermined constant which is determined in the next step.

Referring to the last two equations of (26) we find

$$
p_{4}^{\prime}=\frac{8}{2} c_{2}^{(2)} t^{2}+c_{1}^{(4)} t+c_{2}^{(4)}, \quad w_{4}^{\prime}=c_{3}^{(4)} t+c_{4}^{(4)} .
$$

By conditions $(b)$ and $(c)$ we have

$$
c_{2}^{(2)}=c_{1}^{(4)}=c_{3}^{(4)}=c_{4}^{(4)}=0 .
$$


Therefore $p_{2}^{\prime}$, equation (24), is zero. Similarly $p_{3}^{\prime}$ can be shown to be zero. Consider the equations which define $p_{6}^{\prime}$ and $w_{6}^{\prime}$. They are

$$
\begin{aligned}
& \frac{d^{2} p_{6}^{\prime}}{d \tau^{2}}=3 p_{4}^{\prime}-\frac{E M N_{2}}{2(E+M \bar{L})^{2}}-\frac{3 E M N_{2}}{2(E+M)^{2}} \cos 2 \tau, \\
& \frac{d^{2} w_{6}^{\prime}}{d \tau^{2}}=\frac{3 E M N_{2}}{2(E+M)^{2}} \sin 2 \tau,
\end{aligned}
$$

where $p_{4}^{\prime}=c_{2}^{\prime(4)}$. In order that $p_{6}^{\prime}$ may be periodic the constant part of the right member of the first of these equations must vanish. Therefore

$$
p_{4}^{\prime}=c_{2}^{(4)}=\frac{E M N_{2}}{6(E+M)^{2}} .
$$

Then it is found, after imposing condition (c), that

$$
p_{6}^{\prime}=c_{i}^{(6)}+\frac{3 E M N_{2}}{8(E+M)^{2}} \cos 2 \tau, \quad w_{6}^{\prime}=-\frac{3 E M N_{2}}{8(E+M)^{2}} \sin 2 \tau .
$$

We shall now develop general formulas which will obviate the necessity of integrating the equations at each step. Suppose $p_{2}, \ldots, p_{i-1}, w_{2}, \ldots, w_{i-1}$, $p_{2}^{\prime}, \cdots, p_{i-1}^{\prime}, w_{2}^{\prime}, \cdots, w_{i-1}^{\prime}$ have been computed. Suppose

$$
\begin{aligned}
& p_{j}=\alpha_{0}^{(j)}+\alpha_{1}^{(j)} \cos \tau+\alpha_{2}^{(j)} \cos 2 \tau+\cdots+\alpha_{j}^{(j)} \cos j \tau, \\
& w_{j}=\beta_{1}^{(j)} \sin \tau+\beta_{2}^{(j)} \sin 2 \tau+\cdots+\beta_{j}^{(j)} \sin j \tau, \quad(j=2, \cdots, i-1), \\
& p_{j}^{\prime}=\alpha_{0}^{\prime(j)}+\alpha_{1}^{\prime(j)} \cos \tau+\alpha_{2}^{(j)} \cos 2 \tau+\cdots+\alpha_{j}^{(j)} \cos (j-4) \tau, \\
& w_{j}^{\prime}=\beta_{1}^{\prime(j)} \sin \tau+\beta_{2}^{\prime(j)} \sin 2 \tau+\cdots+{\beta_{j-4}^{\prime(j)}} \sin (j-4) \tau .
\end{aligned}
$$

Suppose the coefficients are all uniquely determined except $\alpha_{1}^{(i-1)}, \beta_{1}^{(i-1)}$, which we shall suppose carry an undetermined constant linearly, and $\alpha_{0}^{(i-2)}$ and $\alpha_{0}^{(i-1)}$, which we shall suppose are so far arbitrary. The problem is to find the coef. ficients in the expressions for $p_{i}, w_{i}, p_{i}^{\prime}$ and $w_{i}^{\prime}$, and to determine $\alpha_{1}^{(i-1)}, \beta_{1}^{(i-1)}$, $\alpha_{0}^{\prime(i-2)}$ and $\alpha_{0}^{\prime(i-1)}$.

Consider the differential equations for the determination of $p_{i}, w_{i}, p_{i}^{\prime}$ and $w_{i}^{\prime}$. The left members of the differential equations are of the same form for all values of $i$ beyond 1 , for example, as given in (26). The right members are polynomials in $p_{1}, \cdots, p_{i-1}, w_{1}, \cdots, w_{i-1}, p_{1}^{\prime}, \cdots, p_{i-1}^{\prime}, w_{1}^{\prime}, \cdots, w_{i-1}^{\prime}$, and linear in $\cos j \tau$ and $\sin j \tau$. In any term which is independent of $\cos j \tau$ and $\sin j \tau$ the sum of the products of the exponents and subscripts cannot exceed $i$. In any term which involves $\cos j \tau$ or $\sin j \tau$ the sum of the products of the exponents and subscripts cannot exceed $i-j$. It follows from the way in which $p, w, p^{\prime}, w^{\prime}$ 
and $\tau$ enter in the differential equations (9) that the right members of the first and third equations which determine $p_{i}, w_{i}, p_{i}^{\prime}$ and $w_{i}^{\prime}$ are even in the $d p_{j} / d \tau$, $w_{j}, d p_{j}^{\prime} / d \tau, w_{j}^{\prime}$ and $\tau$ taken together, and that the second and fourth equations are odd in the same quantities. Hence it follows from the forms of equations (31) that when the values are substituted for the $p_{j}, w_{j}, p_{j}^{\prime}$ and $w_{j}^{\prime}$, the right mem' $>\mathrm{s}$ of the first and third equations will be sums of cosines of multiples of $\tau$, and the right members of the second and fourth equations sums of sines of multiples of $\tau$.

It is necessary to find how $p_{i-1}, w_{i-1}$, and $p_{i-2}^{\prime}$ enter in the right members of the differential equations which determine $p_{i}, w_{i}, p_{i}^{\prime}$, and $w_{i}^{\prime}$. It is seen in the special case (26), and it can be shown in general from equations (9), that the first equation contains the terms $6 p_{i-1}$ and $2 d w_{i-1} / d \tau$, the second $-2 d p_{i-1} / d \tau$, and the third $3 p_{i-2}^{\prime}$. These are the only terms that contain coefficients which are not completely determined. Hence the equations which determine $p_{i}, w_{i}, p_{i}^{\prime}$, and $w_{i}^{\prime}$ have the form

$$
\begin{aligned}
& \frac{d^{2} p_{i}}{d \tau^{2}}-3 p_{i}-2 \frac{d w_{i}}{d \tau}=A_{0}^{(i)}+\left(6 \alpha_{i}^{(i-1)}+2 \beta_{1}^{(i-1)}+A_{1}^{(i)}\right) \cos \tau \\
& +A_{2}^{(i)} \cos 2 \tau+\cdots+A_{i}^{(i)} \cos i \tau, \\
& \frac{d^{2} w_{i}}{d \tau^{2}}+2 \frac{d p_{i}}{d \tau}=\left(2 \alpha_{1}^{(i-1)}+B_{1}^{(i)}\right) \sin \tau+B_{2}^{(i)} \sin 2 \tau+\cdots+B_{i}^{(i)} \sin i \tau, \\
& \frac{d^{2} p_{i}^{\prime}}{d \tau^{2}}=\left(3 \alpha_{0}^{(i-}+A_{0}^{\prime(i)}\right)+A_{1}^{\prime(i)} \cos \tau+\cdots+A_{i-4}^{(i)} \cos (i-4) \tau, \\
& \frac{d^{2} w_{i}^{\prime}}{d \tau^{2}}=B_{1}^{\prime(i)} \sin \tau+\cdots+B_{i-4}^{\prime(i)} \sin (i-4) \tau,
\end{aligned}
$$

where the $A_{j}^{(i)}, B_{j}^{(i)}, A_{j}^{(i)}$, and ${B_{i}^{\prime(i)}}^{(2 r e}$ known numbers depending upon the coefficients of $p_{2}, \cdots, p_{i-1}, v_{2}, \cdots, w_{i-1}, p_{2}^{\prime}, \cdots, p_{i-1}^{\prime}, w_{2}^{\prime}, \cdots, w_{i-1}^{\prime}$. The constants $\alpha_{1}^{(i-1)}, \beta_{1}^{(i-1)}$ and $\alpha_{0}^{(i-2)}$ are as yet undeternined.

The solutions of (32) are to be periodic, and $d p_{i} / d \tau, w_{i}, d p_{i}^{\prime} / d \tau$, and $w_{i}^{\prime}$ must vanish at $\tau=0$. Therefore they must have the form

$$
\begin{aligned}
& p_{i}=x_{0}^{(i)}+\alpha_{1}^{(i)} \cos \tau+\alpha_{2}^{(i)} \cos 2 \tau+\cdots+\alpha_{i}^{(i)} \cos i \tau, \\
& w_{i}=\beta_{1}^{(i)} \sin \tau+\beta_{2}^{(i)} \sin 2 \tau+\cdots+\beta_{i}^{(i)} \sin i \tau, \\
& p_{i}^{\prime}=\alpha_{0}^{\prime(i)}+\alpha_{1}^{\prime(i)} \cos \tau+\alpha_{2}^{\prime(i)} \cos 2 \tau+\cdots+\alpha_{i-4}^{\prime(i)} \cos (i-\dot{4}) \tau, \\
& w_{i}^{\prime}=\beta_{1}^{\prime(i)} \sin \tau+{\beta_{2}^{\prime(i)}}^{\prime} \sin 2 \tau+\cdots+\beta_{i-4}^{\prime(i)} \sin (i-4) \tau .
\end{aligned}
$$

The $\alpha_{j}^{(i)}, \beta_{j}^{(i)}, \alpha_{j}^{\prime(i)}$, and $\beta_{j}^{(i)}$ are to be determined in terms of the $A_{j}^{(i)}, B_{j}^{(i)}, A_{j}^{\prime(i)}, B_{j}^{\prime(i)}$, and the new constants of integration.

Consider first the third and fourth equations of (32). In order that $p_{i}^{\prime}$ may be periodic we must impose the condition 


$$
\alpha_{0}^{(i-2)}=-\frac{1}{3} A_{0}^{\prime(i)} .
$$

Then imposing conditions $(b)$ and $(c)$ we have

$$
\begin{aligned}
& \alpha_{0}^{(i)}=\text { constant undetermined until computation of } p_{i+2}, \\
& \alpha_{j}^{(i)}=-\frac{1}{j^{2}} A_{j}^{\prime(i)} \\
& {\beta_{j}^{\prime(i)}}^{\prime(i)}=-\frac{1}{j^{2}} B_{j}^{\prime(i)} .
\end{aligned}
$$

Now consider the first two equations of (32). Integrating the second we have

(35) $\frac{d w_{i}}{d \tau}=c_{1}^{(i)}-2 p_{i}-\left(2 \alpha_{1}^{(i-1)}+B_{1}^{(i)}\right) \cos \tau-\frac{1}{2} B_{2}^{(i)} \cos 2 \tau-\cdots-\frac{1}{i} B_{i}^{(i)} \cos i \tau$.

Substituting this expression for $d w_{i} / d \tau$ in the first of (32), we find

$$
\begin{aligned}
\frac{d^{2}}{d} \frac{p_{i}}{\tau^{2}}+p_{i} & =\left[A_{1}^{(i)}+2 c_{1}^{(i)}\right]+\left[A_{1}^{(i)}-2 B_{1}^{(i)}+2 \alpha_{1}^{(i-1)}+2 \beta_{1}^{(i-1)}\right] \cos \tau \\
& +\left[A_{2}^{(i)}-\frac{2}{2} B_{2}^{(i)}\right] \cos 2 \tau+\cdots+\left[A_{j}^{(i)}-\frac{2}{j} B_{j}^{(i)}\right] \cos j \tau+\cdots \\
& +\left[A_{i}^{(i)}+\frac{2}{i} B_{i}^{(i)}\right] \cos i \tau .
\end{aligned}
$$

In order that the solution of this equation may be periodic [condition (b)] we must impose the condition

$$
A_{1}^{(i)}-2 B_{1}^{(i)}+2 \alpha_{1}^{(i-1)}+2 \beta_{1}^{(i-1)}=0 .
$$

Then the most general solution of (36) satisfying condition $(c)\left[d p_{i} / d \tau=0\right.$ at $\tau=0]$ is

$$
\begin{aligned}
p_{i}=\left[A_{0}^{(i)}+2 c_{1}^{(i)}\right]+\alpha_{1}^{(i)} \cos \tau-\frac{\left[2 A_{2}^{(i)}-2 B_{2}^{(i)}\right]}{2\left(2^{2}-1\right)} \cos 2 \tau-\cdots \\
-\frac{\left[j A_{j}^{(i)}-2 B_{j}^{(i)}\right]}{j\left(j^{2}-1\right)} \cos j \tau-\cdots-\frac{\left[i A_{i}^{(i)}-2 B_{i}^{(i)}\right]}{i\left(i^{2}-1\right)} \cos i \tau .
\end{aligned}
$$

Substituting this value of $p_{i}$ in (35), we get

$$
\begin{aligned}
\frac{d w_{i}}{d \tau}=- & {\left[2 A_{0}^{(i)}+3 c_{1}^{(i)}\right]-\left[B_{1}^{(i)}+2 \alpha_{1}^{(i)}+2 \alpha_{1}^{(i-1)}\right] \cos \tau+\frac{\left[4 A_{2}^{(i)}-7 B_{2}^{(i)}\right]}{2\left(2^{2}-1\right)} \cos 2 \tau+\cdots } \\
& +\frac{\left[2 j A_{j}^{(i)}-\left(j^{2}+3\right) B_{j}^{(i)}\right]}{j\left(j^{2}-1\right)} \cos j \tau+\cdots+\frac{\left[2 i A_{i}^{(i)}-\left(i^{2}+3\right) B_{i}^{(i)}\right]}{i\left(i^{2}-1\right)} \cos i \tau .
\end{aligned}
$$


In order that the solution of this equation may be periodic we must impose the condition

$$
2 A_{0}^{(i)}+3 c_{1}^{(i)}=0
$$

which determines $c_{1}^{(i)}$ uniquely. Substituting in the first term in the right member of (38), we find $\alpha_{0}^{(i)}=A_{0}^{(i)}+2 c_{1}^{(i)}=-\frac{1}{3} A_{0}^{(i)}$. Then the most general solution of the equation for $w_{i}$, vanishing at $\tau=0$, is

$$
\begin{aligned}
w_{i} & =-\left[B_{1}^{(i)}+2 \alpha_{1}^{(i)}+2 \alpha_{1}^{(i-1)}\right] \sin \tau+\frac{\left[4 A_{2}^{(i)}-7 B_{2}^{(i)}\right]}{12} \sin 2 \tau+\cdots \\
& +\frac{\left[2 j A_{j}^{(i)}-\left(j^{2}+3\right) B_{j}^{(i)}\right]}{j^{2}\left(j^{2}-1\right)} \sin j \tau+\cdots+\frac{\left[2 i A_{i}^{(i)}-\left(i^{2}+3\right) B_{i}^{(i)}\right]}{i^{2}\left(i^{2}-1\right)} \sin i \tau .
\end{aligned}
$$

In the notation adopted in (33) the coefficient of $\sin \tau$ in equation (40) is $\beta_{1}^{(i)}$. Since the form is general we have

$$
\beta_{1}^{(i-1)}=-\left[B_{1}^{(i-1)}+2 \alpha_{1}^{(i-1)}+2 \alpha_{1}^{(i-2)}\right] .
$$

This equation used with (37) determines $\alpha_{1}^{(i-1)}$ and $\beta_{1}^{(i-1)}$ in terms of known quantities. Hence the general expressions for the coefficients of (33) are

$$
\begin{array}{rlr}
\alpha_{0}^{(i)} & =-\frac{1}{3} A_{0}^{(i)} & (i=2, \cdots, \infty), \\
\alpha_{1}^{(i-1)} & =\frac{1}{2}\left[A_{1}^{(i)}-2 B_{1}^{(i)}\right]-\left[B_{1}^{(i-1)}+2 \alpha_{1}^{(i-2)}\right], & \\
\beta_{1}^{(i-1)} & =-\left[A_{1}^{(i)}-2 B_{1}^{(i)}\right]+\left[B_{1}^{(i-1)}+2 \alpha_{1}^{(i-2)}\right], & \\
\alpha_{j}^{(i)} & =-\frac{\left[j A_{j}^{(i)}-2 B_{j}^{(i)}\right]}{j\left(j^{2}-1\right)} & (j=2, \cdots, i), \\
\beta_{j}^{(i)} & =\frac{\left[2 j A_{j}^{(i)}-\left(j^{2}+3\right) B_{j}^{(i)}\right]}{j^{2}\left(j^{2}-1\right)} & (j=2, \cdots, i), \\
\alpha_{0}^{\prime(i-2)} & =-\frac{1}{3} A_{0}^{\prime(i)} & (i=6, \cdots, \infty), \\
\alpha_{j}^{(i)} & =-\frac{1}{j^{2}} A_{j}^{\prime(i)} & (j=1, \cdots, i-4), \\
\beta_{j}^{\prime(i)} & =-\frac{1}{j^{2}} B_{j}^{\prime(i)} &
\end{array}
$$

The simplicity of the computation from these formulas and the expansions (22) will be illustrated by the computation of $\alpha_{j}^{(4)}$ and $\beta_{j}^{(4)}$. 
TABLe I.

\begin{tabular}{|c|c|c|c|c|c|}
\hline Term from (22). & Constant. & $\cos \tau$ & $\cos 2 \tau$ & $\cos 3 \tau$ & $\cos 4 \tau$ \\
\hline$+2 \frac{d w_{3}}{d \tau}$ & 0 & $-2 \beta_{1}^{(3)}$ & $+\frac{26}{3} \eta$ & 0 & 0 \\
$+2 p_{2} \frac{d w_{2}}{d \tau}$ & $-\frac{11}{4} \eta^{2}$ & 0 & $-\frac{1}{1} \frac{1}{2} \eta^{2}$ & 0 & $-\frac{11}{4} \eta^{2}$ \\
$+6 p_{3}$ & $+2 \eta$ & $+6 a_{1}^{(3)}$ & $-7 \eta$ & 0 & 0 \\
$+\left(\frac{d w_{2}}{d \tau}\right)^{2}$ & $+\frac{121}{32} \eta^{2}$ & 0 & 0 & 0 & $+\frac{121}{3} \eta^{2}$ \\
$+3 p_{2}$ & $-\frac{1}{2} \eta$ & 0 & $-3 \eta$ & 0 & 0 \\
$-3 p_{2}^{2}$ & $-\frac{19}{2} \eta^{2}$ & 0 & $-\eta^{2}$ & 0 & $-\frac{3}{2} \eta^{2}$ \\
$+\frac{1}{2} \eta p_{2}(1+3 \cos 2 \tau)$ & $-\frac{5}{6} \eta^{2}$ & 0 & $-\frac{3}{4} \eta^{2}$ & 0 & $-\frac{3}{4} \eta^{2}$ \\
$-3 \eta w_{2} \sin 2 \tau$ & $-\frac{3}{1} \frac{3}{6} \eta^{2}$ & 0 & 0 & 0 & $+\frac{83}{16} \eta^{2}$ \\
$+\frac{3}{8} \eta M_{1}(3 \cos \tau+5 \cos 3 \tau)$ & 0 & $+\frac{9}{8} \eta M_{1}$ & 0 & $+\frac{15}{8} \eta M_{1}$ & 0 \\
\hline$A_{f}^{(4)}$ & $+\frac{8}{2} \eta-\frac{33}{96} \eta^{2}$ & $+\frac{9}{8} \eta M_{1}$ & $-\frac{4}{3} \eta-\frac{8}{8} \eta^{2}$ & $+\frac{15}{8} \eta M_{1}$ & $+\frac{27}{82} \eta^{2}$ \\
\hline
\end{tabular}

Table II.

\begin{tabular}{|c|c|c|c|c|}
\hline Term from (22). & $\sin \tau$ & $\sin 2 \tau$ & $\sin 3 \tau$ & $\sin 4 \tau$ \\
\hline$-p_{2} \frac{d^{2} w_{2}}{d \tau^{2}}$ & 0 & $-\frac{11}{12} \eta^{2}$ & 0 & $-\frac{11}{4} \eta^{2}$ \\
$-2 \frac{d p_{3}}{d \tau}$ & $+2 \alpha_{1}^{(3)}$ & $-\frac{14}{3} \eta$ & 0 & 0 \\
$-2 \frac{d p_{2}}{d \tau} \frac{d w_{2}}{d \tau}$ & 0 & 0 & 0 & $-\frac{11}{2} \eta^{2}$ \\
$-\frac{8}{2} \eta p_{2} \sin 2 \tau$ & 0 & $+\frac{1}{4} \eta^{2}$ & 0 & $+\frac{3}{4} \eta^{2}$ \\
$-3 \eta w_{2} \cos 2 \tau$ & 0 & 0 & 0 & $-\frac{3}{16} \eta^{2}$ \\
$-\frac{3}{8} \eta M_{1}(\sin \tau+5 \sin 3 \tau)$ & $-\frac{3}{8} \eta M_{1}$ & 0 & $-\frac{15}{8} \eta M_{1}$ & 0 \\
\hline$B_{j}^{(4)}$ & $-\frac{3}{8} \eta M_{1}$ & $-\frac{14}{8} \eta-\frac{2}{3} \eta^{2}$ & $-\frac{15}{8} \eta M_{1}$ & $-\frac{153}{16} \eta^{2}$ \\
\hline
\end{tabular}

From these values of $A_{j}^{(4)}$ and $B_{j}^{(4)}\left(B_{j}^{(3)}\right.$ and $\alpha_{1}^{(2)}$ are zero $)$ it is found by equations (42) that

$$
\begin{array}{ll}
\alpha_{0}^{(4)}=-\frac{\eta}{2}+\frac{3}{2} \frac{81}{8} \eta^{2}, & \\
\alpha_{1}^{(3)}=+\frac{1}{1} \frac{5}{6} \eta M_{1}, & \beta_{1}^{(3)}=-\frac{15}{8} \eta M_{1}, \\
\alpha_{2}^{(4)}=-\frac{10}{9} \eta+\frac{2}{3} \eta^{2}, & \beta_{2}^{(4)}=+\frac{4}{1} \frac{1}{8} \eta-\frac{1}{2} \eta^{2}, \\
\alpha_{3}^{(4)}=-\frac{25}{6} \eta M_{1}, & \beta_{3}^{(4)}=+\frac{1}{3} \frac{5}{2} \eta M_{1}, \\
\alpha_{4}^{(4)}=-\frac{3}{8} \eta^{2}, & \beta_{4}^{(4)}=+\frac{2}{2} \frac{1}{6} \frac{1}{6} \eta^{2} .
\end{array}
$$

As many succeeding $\alpha_{j}^{(i)}$ and $\beta_{j}^{(i)}$ as may be desired may be computed in precisely 
the same way. The operations are reduced to systematic routine, and the work consists almost wholly in multiplying fractions in constructing the tables, and in adaing fractions in getting the $A_{j}^{(i)}, B_{j}^{(i)}, A_{j}^{(i)}$ and $B_{j}^{\prime(i)}$.

The differential equations admit two integrals of which no use has been made. They may be used to derive new formulas for the computation of the $\alpha_{j}^{(i)}, \beta_{j}^{(i)}$, $\alpha_{j}^{\prime(i)}$, and $\beta_{i}^{\prime(i)}$, or to verify the results obtained by (42). The principal applications occur when the mass of $M$ is infinitesimal, or at the most so small that its effect on the motion of $S$ around the center of gravity of $E$ and $M$ is insensible. Consequently, to abbreviate the discussion we shall assume that $S$ moves in an undisturbed circle around the center of gravity of $E$ and $M$. Then the integral of areas disappears and the vis viva integral becomes $\mathrm{J}_{A C O B I}$ 's integral:

$$
\begin{gathered}
\left(\frac{d p}{d \tau}\right)^{2}+(1+p)^{2}\left(1+\frac{d w}{d \tau}\right)^{2}-m^{2}(1+p)^{2}-\frac{2(1+m)^{2}}{1+p} \\
-2 m^{2} \eta\left(\frac{a^{\prime}}{a}\right)^{2}\left\{\frac{1}{\sqrt{1-2 \frac{a}{a^{\prime}}(1+p) \cos (\tau+w)+\left(\frac{a}{a^{\prime}}\right)(1+p)^{2}}}\right. \\
\left.-\frac{a}{a}(1+p) \cos (\tau+w)\right\}=C,
\end{gathered}
$$

where $C$ is the Jacobian constant of integration.

In the periodic solution $p$ and $w$ are developed as power series in $m$, and hence we may write the integral for this solution in the form

$$
\begin{aligned}
F_{0}+F_{1}\left(p_{1}, \frac{d p_{1}}{d \tau}, w_{1}, \frac{d w_{1}}{d \tau} ; \tau\right) m \\
\quad+F_{2}\left(p_{1}, p_{2}, \frac{d p_{1}}{d \tau}, \frac{d p_{2}}{d \tau}, \ldots, \frac{d w_{2}}{d \tau} ; \tau\right) m^{2}+\cdots=C .
\end{aligned}
$$

Since this integral converges for all values of $m$ sufficiently small, it follows that each $F_{i}$ is separately constant. In $F_{i}$ the sum of the products of the exponents and subscripts of the factors of any term independent of $\cos j \tau$ cannot exceed $i$; and in any term involving $\cos j \tau$ the sum cannot exceed $i-j$. The function $F$ is even in $d p_{j} / d \tau, w_{j}$ and $\tau$ and therefore each $F_{i}$ has the form

$$
F_{i}=\gamma_{0}^{(i)}+\gamma_{1}^{(i)} \cos \tau+\cdots+\gamma_{j}^{(i)} \cos j \tau+\cdots+\gamma_{i}^{(i)} \cos i \tau .
$$

Since every $F_{i}$ is identically constant in $\tau$ it follows that

$$
\gamma_{j}^{(i)}=0 \quad(i=1, \cdots, \infty ; j=1, \cdots, i) .
$$

The fulfillment of these relations, which are functions of $\alpha_{j}^{(2)}, \ldots, \alpha_{j}^{(i)}, \beta_{j}^{(2)}, \ldots, \beta_{j}^{(i)}$ $(j=0, \cdots, i)$, serves as a complete check on the expansions and the computations. 
The integral is applied by first expanding the separate terms of (44) as power series in $m$. Thus we have the expansions

$$
\begin{aligned}
& \left(\frac{d p}{d \tau}\right)^{2}=\left(\frac{d p_{2}}{d \tau}\right)^{2} m^{4}+2 \frac{d p_{2}}{d \tau} \frac{d p_{3}}{d \tau} m^{5}+\cdots, \\
& (1+p)^{2}\left(1+\frac{d w}{d \tau}\right)^{2}=1+2\left[p_{2}+\frac{d w_{2}}{d \tau}\right] m^{2}+2\left[p_{3}+\frac{d w_{3}}{d \tau}\right] m^{3} \\
& \quad+\left[2 p_{4}+p_{2}^{2}+2 \frac{d w_{4}}{d \tau}+\left(\frac{d w_{2}}{d \tau}\right)^{2}+4 p_{2} \frac{d w_{2}}{d \tau}\right] m^{4}+\cdots, \\
& -m^{2}(1+p)^{2}=-m^{2}-2 p_{2} m^{4}+\cdots, \\
& -\frac{2(1+m)^{2}}{1+p}=-2-4 m-2\left[1-p_{2}\right] m^{2}-2\left[2 p_{2}+p_{3}\right] m^{3} \\
& \quad-2\left[p_{4}-p_{2}^{2}+2 p_{3}+p_{2}\right] m^{4}+\cdots, \\
& -m^{2} \eta\left(\frac{a^{\prime}}{a}\right)^{2}\left\{\left[1-2 \frac{a}{a^{\prime}}(1+p) \cos (\tau+w)+\left(\frac{a}{a^{\prime}}\right)^{2}(1+p)^{2}\right]^{-\frac{1}{1}}\right. \\
& \left.\quad-\frac{a}{a^{\prime}}(1+p) \cos (\tau+w)\right\}=-\frac{1}{2} \eta[1+3 \cos 2 \tau] m^{2} \\
& +\left[-\eta p_{2}(1+3 \cos 2 \tau)+3 \eta w_{2} \sin 2 \tau-\frac{1}{4} M_{1} \eta(3 \cos \tau+5 \cos 3 \tau)\right] m^{4}+\cdots
\end{aligned}
$$

It can be shown from these functions that in general $F_{i}$ contains the terms $4 p_{i}, 2 d w_{i} / d \tau, 4 p_{i-1}$, and terms with lower subscripts. Suppose the coefficients of $p_{2}, \ldots, p_{i-2}, w_{2}, \ldots, w_{i-2}$ are entirely known, and that the coefficients of $p_{i-1}$, and $w_{i-1}$ are all known except $\alpha_{1}^{(i-1)}$ and $\beta_{1}^{(i-1)}$. Let in general the known part of $\gamma_{j}^{(i)}$ be $C_{j}^{(i)}$. Then the conditions (46) become

$$
\begin{aligned}
4 \alpha_{1}^{(i)}+2 \beta_{1}^{(i)}+4 \alpha_{1}^{(i-1)}+C_{1}^{(i)}=0 & (i=2, \cdots, \infty) . \\
4 \alpha_{j}^{(i)}+2 j \beta_{j}^{(i)}+C_{j}^{(i)}=0 & (j=2, \cdots, i),
\end{aligned}
$$

From equations (42) it is found that

$$
\begin{aligned}
4 \alpha_{1}^{(i)}+2 \beta_{1}^{(i)}+4 \alpha_{1}^{(i-1)}+2 B_{1}^{(i)}=0 & (i=2, \cdots, \infty), \\
4 \alpha_{j}^{(i)}+2 j \beta_{j}^{(i)}+\frac{2}{j} B_{j}^{(i)}=0 & (j=2, \cdots, i) .
\end{aligned}
$$

The comparison of these equations with (48) shows that

$$
C_{j}^{(i)}-\frac{2}{j} B_{j}^{(i)}=0 \quad(i=2, \cdots, \infty ; j=1, \cdots, i) .
$$

When the $B_{j}^{(i)}$ have been eliminated from equations (42) by means of these relations, the $\alpha_{j}^{(i)}$ and $\beta_{j}^{(i)}$ are expressed in terms of the $A_{j}^{(i)}$ and $C_{j}^{(i)}$ by the equations 


$$
\begin{array}{rlr}
\alpha_{0}^{(i)} & =-\frac{1}{3} A_{0}^{(i)} & (i=2, \cdots, \infty), \\
\alpha_{1}^{(i-1)} & =\frac{1}{2}\left[A_{1}^{(i)}-C_{1}^{(i)}\right]-\left[\frac{1}{2} C_{1}^{(i-1)}+2 \alpha_{1}^{(i-2)}\right], & \\
\beta_{1}^{(i-1)} & =-\left[A_{1}^{(i)}-C_{1}^{(i)}\right]+\left[\frac{1}{2} C_{1}^{(i-1)}+2 \alpha_{1}^{(i-2)}\right], & \\
\alpha_{j}^{(i)} & =-\frac{\left[A_{j}^{(i)}-C_{j}^{(i)}\right]}{j^{2}-1} & \\
\beta_{j}^{(i)} & =\frac{\left[4 A_{j}^{(i)}-\left(j^{2}+3\right) C_{j}^{(i)}\right]}{2 j\left(j^{2}-1\right)} . &
\end{array}
$$

Therefore in case the orbit of $S$ is a circle around the center of gravity of $E$ and $M$ the second differential equation, which involves the second derivative of $w$, may be entirely neglected in the determination of the coefficients of the periodic solution, and $J_{A C O B I}$ 's integral used in its place.* The more convenient process is to be employed. In every case there are more terms in the table for the $C_{j}^{(i)}$ than for the $B_{j}^{(i)}$, but a large proportion of the former are the same as those occurring in the table for the $A_{j}^{(i)}$ except for numerical factors. For

\begin{tabular}{|c|c|c|c|c|c|}
\hline Term from (47). & Constaut. & $\cos \tau$ & $\cos 2 \tau$ & $\cos \tau$ & $\cos 4 \tau$ \\
\hline$+\left(\frac{d p_{2}}{d \tau}\right)^{2}$ & $+2 \eta^{2}$ & 0 & 0 & 0 & $-2 \eta^{2}$ \\
\hline$+4 p$ & $+4 a_{10}^{(4)}$ & $+4 a_{1}^{(4)}$ & $+4 a_{2}^{(4)}$ & $+4 a_{3}^{(4)}$ & $+4 a_{t}^{(4)}$ \\
\hline$-p_{2}^{2}$ & $-\frac{19}{3} \frac{9}{6} \eta^{2}$ & 0 & $-\frac{1}{3} \eta^{2}$ & 0 & $-\frac{1}{2} \eta^{2}$ \\
\hline$+2 \frac{d w_{4}}{d \tau}$ & 0 & $+2 \beta_{1}^{(4)}$ & $+4 \beta_{:}^{(4)}$ & $+6 \beta_{3}^{(4)}$ & $+8 \beta_{4}^{(4)}$ \\
\hline$+\left(\frac{d w_{2}}{d \tau}\right)^{2}$ & $+\frac{12}{32} \eta^{2}$ & 0 & 0 & 0 & $+\frac{121}{32} \eta^{2}$ \\
\hline$+4 p_{2} \frac{d w_{2}}{d \tau}$ & $-\frac{11}{2} \eta^{2}$ & 0 & $-\frac{11}{6} \eta^{2}$ & 0 & $-\frac{11}{1} \eta^{2}$ \\
\hline$-\eta p_{2}$ & $+\frac{1}{6} \eta^{2}$ & 0 & $+\eta^{2}$ & 0 & 0 \\
\hline$+4 p_{3}$ & $+\frac{4}{3} \eta$ & $4 \boldsymbol{a}_{!}^{(3)}$ & $-\frac{14}{3} \eta$ & 0 & 0 \\
\hline$-3 \eta p_{2} \cos 2 \tau$ & $+\frac{3}{2} \eta^{2}$ & 0 & $+\frac{1}{2} \eta^{2}$ & 0 & $+\frac{6}{2} \eta^{2}$ \\
\hline$+3 \eta w_{2} \sin 2 \tau$ & $+\frac{3.3}{16} \eta^{2}$ & 0 & 0 & 0 & $-\frac{33}{16} \eta^{2}$ \\
\hline$-\frac{1}{4} M_{1} \eta(3 \cos \tau+5 \cos 3 \tau)$ & 0 & $-\frac{3}{4} M_{1} \eta$ & 0 & $-\frac{5}{4} d \|_{1} \eta$ & 0 \\
\hline$C_{j}^{(4)}$ & $\mid+\frac{4}{3} \eta+\frac{251}{72} \eta^{2}$ & $-3 M_{1} M$ & $-\frac{14}{3} \eta-\frac{2}{3} \eta^{2}$ & - & $-1.3 \eta^{2}$ \\
\hline
\end{tabular}
this reason the $\alpha_{j}^{(i)}$ and $\beta_{j}^{(2)}$ are more conveniently found from equations (50) than they are from equations (42), but then the advantage of having the integral as a check is lost. In the computations given in this paper the coefficients were obtained by (42), and the integral was used as a check.

The use of the integral will be illustrated in computing the coefficients of $\mathrm{m}^{4}$.

TABLE III.

* Hill used the integral to eliminate the most troublesome expression arising in the differential equations in bis method of treatment. Loc. cit., p. 13:. 
With these values of the $C_{j}^{(4)}$ and the $A_{j}^{(4)}$ of table I, equations (50) lead to the results of (43); or, when the values from (43) are substituted in table III, the relations $(46)$ are satisfied.

Suppose the $\alpha_{j}^{(i)}$ and $\beta_{j}^{(i)}$ are computed by (42) and that the integral is used as a check. It follows from (48) and (49) that at the $i$ th step the check does not cover the $A_{j}^{(i)}$. That is, an error might exist in any $A_{j}^{(i)}$ without its being revealed by the check formula until the $(i+1)$ st step. However, there is the check for the $A_{j}^{(i)}$ and $C_{j}^{(i)}$ that the sums of the coefficients in every line of their tables must equal the values of the functions in the first columns at $\tau=0$, and the sums of the $A_{j}^{(i)}$ and $C_{j}^{(i)}$ (with respect to $j$ ) must equal the sums of their respective first columns at $\tau=0$.

\section{Appilcations to the lunar theory.}

The differential equations and their solutions have been developed in such a form as to be immediately applicable to the Lunar Theory, provided we assume that its motion is periodic of the type under consideration. It is only necessary to find the order of magnitude of the parameters involved so as to know how far the developments must be carried to meet the needs of observers. Observations have given for the Lunar Theory the following approximate numerical relations :

$$
\begin{aligned}
& n=13.4 n^{\prime}, \text { whence } m=\frac{n^{\prime}}{n-n^{\prime}}=\frac{1}{12.4}, \\
& E=80 M, \\
& S=330,000 E, \text { whence } \eta=\frac{S}{S+E+M}=\frac{1}{1+\frac{1}{300,000}}, \\
& \frac{a}{a^{\prime}}={ }_{4}^{\frac{1}{0} \overline{0}}, \text { whence } M_{1}=N_{1}=\frac{3}{8}, M_{2}=N_{2}=\frac{1}{7}, M_{3}=N_{3}=\frac{1}{18} .
\end{aligned}
$$

Consider first the expressions for $p^{\prime}$ and $w^{\prime}$. The term of lowest degree in $p^{\prime}$ is, by (29), $p^{\prime}=p_{4}^{\prime} m^{4}=E M N_{2} m^{4} / 6(E+M)^{2}$. .It follows from (51) that $p_{4}^{\prime} m^{4}=1 / 80,000,000$. That is, since $r^{\prime}=a^{\prime}\left(1+p^{\prime}\right)$, the average distance of the sun from the center of gravity of the earth and moon is about 1.2 miles greater than it would be if it were not disturbed by the moon. 'The first variable terms in $p^{\prime}$ and $w^{\prime}$ are, by (30),

$$
p_{6}^{\prime} m^{6}=+\frac{3}{8} \frac{E M N_{2} m^{6}}{(E+M)^{2}} \cos 2 \tau, \quad w_{6}^{\prime} m^{6}=-\frac{3}{8} \frac{E M N_{2} m^{6}}{(E+M)^{2}} \sin 2 \tau
$$

Therefore, with respect to the center of gravity of the earth and moon as an origin, the sun describes in a period of one half a synodical month in the retrograde direction a nearly circular orbit of radius 95 feet around a point whose distance is approximately $a^{\prime}\left(1+p_{4}^{\prime} m^{4}+p_{5}^{\prime} m^{5}+p_{6}^{\prime} m^{6}\right)$, and which revolves with the mean angular motion of the sun. 
The coördinates of the sun, $p^{\prime}$ and $w^{\prime}$, enter in the right members of the first two equations of (9). It has been shown how slightly they vary because of perturbations by the moon. The reciprocal action of these perturbations upon the moon are still smaller, because where they appear in the differential equations they are multiplied by $m^{2}$. If we regard $M N_{2} /(E+M)$ as of the second order in $m$, the perturbations of the moon by the sun due to the fact that the moon has disturbed the sun from a strictly circular orbit are of the 8th order in $m$, and their numerical coefficients are so small that they are absolutely inappreciable observationally. That is, no sensible error is committed in computing the moon's coördinates by assuming that the sun goes around the center of gravity of the earth and moon in an undisturbed orbit.

It is seen from (51) that the constant $\eta$ in the Lunar Theory is very nearly equal to unity. Since the higher terms are much simplified by replacing it by unity while no sensible error is committed, we shall retain it explicitly only up to terms of order 4 in $m$. By applying equations (42) where the $A_{j}^{(i)}$ and $B_{j}^{(i)}$ are defined in (32), it has been found that*

$$
\begin{aligned}
& \frac{r}{a}=-\left[\frac{1}{6}+\cos 2 \tau\right] \eta m^{2}+\left[\frac{1}{\overline{3}}+\frac{15}{16} M_{1} \cos \tau-\frac{7}{6} \cos 2 \tau\right] \eta m^{3}+\left[\frac{331}{32 \cdot 9} \eta-\frac{1}{2}\right. \\
& +\frac{3}{32}(45 \eta-1) M_{1} \cos \tau+\frac{2}{9}(3 \eta-5) \cos 2 \tau-\frac{25}{64} M_{1} \cos 3 \tau \\
& \left.-\frac{3}{8} \eta \cos 4 \tau\right] \eta m^{4}+\left[\frac{181}{16 \cdot 9}+\frac{3145}{256} M_{1} \cos \tau-\frac{19}{2 \cdot 27} \cos 2 \tau+\frac{95}{128} M_{1} \cos 3 \tau\right. \\
& \left.-\frac{497}{32 \cdot 3 \cdot 5} \cos 4 \tau\right] m^{5}+\left[\left(\frac{3175}{32 \cdot 81}+\frac{225}{512} M_{1}^{2}-\frac{3}{16} M_{2}\right)+\frac{56831}{1024} M_{1} \cos \tau\right. \\
& -\left(\frac{10933}{256 \cdot 81}+\frac{225}{512} M_{1}^{2}+\frac{5}{8} M_{2}\right) \cos 2 \tau+\frac{5053}{256 \cdot 3} M_{1} \cos 3 \tau-\left(\frac{4897}{16 \cdot 9 \cdot 25}\right. \\
& \left.\left.+\frac{7}{32} M_{2}\right) \cos 4 \tau-\frac{155}{512} M_{1} \cos 5 \tau-\frac{59}{256} \cos 6 \tau\right] m^{6}+\left[\left(\frac{245}{8 \cdot 27}+\frac{105}{32} M_{1}^{2}\right.\right. \\
& \left.+\frac{3}{8} M_{2}\right)+\left(\frac{910,469}{1024 \cdot 3} M_{1}+\frac{105}{128} M_{3}\right) \cos \tau+\left(\frac{911,671}{512 \cdot 243 \cdot 5}-\frac{8985}{1024} M_{1}^{2}\right. \\
& \left.-\frac{5}{8} M_{2}\right) \cos 2 \tau+\frac{285,283}{4096 \cdot 3} M_{1} \cos 3 \tau-\left(\frac{130,723}{32 \cdot 27 \cdot 125}-\frac{525}{1024} M_{1}^{2}\right. \\
& \left.\left.+\frac{49}{32 \cdot 3 \cdot 5} M_{2}\right) \cos 4 \tau+\frac{625}{1024 \cdot 3} M_{1} \cos 5 \tau-\frac{51853}{512 \cdot 3 \cdot 5 \cdot 7} \cos 6 \tau\right] m^{7}
\end{aligned}
$$

* Mr. W, D. MacMrllan has duplicated the entire computation as a check. Moreover, the integral and other check formulas have been exactly satisfied, and it is not believed that there can be any errors in these series. 


$$
\begin{aligned}
& +\left[\left(\frac{2,228,365}{8192 \cdot 243}+\frac{165,615}{8192} M_{1}^{2}+\frac{53}{32} M_{2}\right)+\left(\frac{228,305,129}{16384 \cdot 9} M_{1}+\frac{1125}{256} M_{1} M_{2}\right.\right. \\
& \left.+\frac{225}{64} M_{3}\right) \cos \tau+\left(\frac{8,581,859}{64 \cdot 729 \cdot 25}-\frac{203,593}{4096} M_{1}^{2}+\frac{1681}{256} M_{2}\right) \cos 2 \tau \\
& +\left(\frac{1,145,073}{4096 \cdot \overline{3}} M_{1}-{ }_{1024}^{245} M_{3}\right) \cos 3 \tau-\left(\frac{8,398,553}{256 \cdot 81 \cdot 625}-\frac{4245}{2048} M_{1}^{2}\right. \\
& \left.+\frac{3241}{64 \cdot 9 \cdot 25} M_{2}\right) \cos 4 \tau+\left(\frac{943,835}{16384 \cdot 9} M_{1}-\frac{147}{1024} M_{3}\right) \cos 5 \tau \\
& -\left(\frac{698,591}{32 \cdot 9 \cdot 25 \cdot 49}+\frac{425}{8192} M_{1}^{2}+\frac{49}{256} M_{2}\right) \cos 6 \tau-\frac{31885}{16384 \cdot 7} M_{1} \cos 7 \tau \\
& \left.-\frac{529}{1024 \cdot 3} \cos 8 \tau\right] m^{8}+\left[\left(-\frac{13,799}{4096 \cdot 9}+\frac{5,765,369}{16384 \cdot 3} M_{1}^{2}+\frac{5}{4 \cdot 3} M_{2}\right)\right. \\
& +\left(\frac{4,337,803,547}{65536 \cdot 9} M_{1}+\frac{7575}{256} M_{1} M_{2}+\frac{64,025}{2048 \cdot 3} M_{3}+\frac{166,875}{32768} M_{1}^{3}\right) \cos \tau \\
& +\left(\frac{1,924,392,199}{512 \cdot 2187 \cdot 125}-\frac{968,633}{512 \cdot 9} M_{1}^{2}+\frac{346,881}{512 \cdot 9 \cdot 5} M_{2}\right) \cos 2 \tau \\
& +\left(\frac{1,018,308,773}{16384 \cdot 27 \cdot 5} M_{1}-\frac{14,445}{8192} M_{1} M_{2}+\frac{3045}{4096} M_{3}+\frac{10,125}{32768} M_{1}^{3}\right) \cos 3 \tau \\
& +\left(\begin{array}{c}
1,092,030,283 \\
1024 \cdot 27 \cdot 3125 \cdot 7
\end{array}-\frac{3,348,655}{16384 \cdot 9 \cdot 5} M_{1}^{2}-\frac{259,997}{64 \cdot 27 \cdot 125} M_{2}\right) \cos 4 \tau \\
& +\left(\frac{264,181,703}{65536 \cdot 27 \cdot 5} M_{1}+\frac{3045}{8192} M_{1} M_{2}-\frac{217}{4096} M_{3}\right) \cos 5 \tau \\
& +\left(-\frac{1,597,008,893}{512 \cdot 27 \cdot 125 \cdot 343}+\frac{62,915}{4096 \cdot 3 \cdot 7} M_{1}^{2}-\frac{3271}{512 \cdot 3 \cdot 5} M_{2}\right) \cos 6 \tau \\
& \left.-\frac{5057}{32768} M_{1} \cos 7 \tau-\frac{5,7 \pi 8,113}{8192 \cdot 3 \cdot 5 \cdot 49} \cos 8 \tau\right] m^{9}+\cdots, \\
& v=(1+m) \tau+\left[\frac{11}{8} \sin 2 \tau\right] \eta m^{2}+\left[-\frac{15}{8} M_{1} \sin \tau+\frac{13}{6} \sin 2 \tau\right] \eta m^{3} \\
& +\left[-{ }_{16}^{3}(45 \eta+7) M_{1} \sin \tau-\frac{1}{18}(9 \eta-41) \sin 2 \tau+\frac{15}{3 g^{2}} M Y_{1} \sin 3 \tau+\frac{201}{256} \sin 4 \tau\right] \eta m^{1} \\
& +\left[-\frac{4151}{128} M M_{1} \sin \tau+\frac{89}{4} 27 \sin 2 \tau-\frac{25}{16} M M_{1} \sin 3 \tau+\frac{2177}{64 \cdot 3 \cdot 5} \sin 4 \tau\right] m^{5}
\end{aligned}
$$


$+\left[-\frac{71,449}{512} M_{1} \sin \tau-\left(\frac{62,911}{2048 \cdot 81}-\frac{1125}{1024} M_{1}^{2}-\frac{25}{32} M_{2}\right) \sin 2 \tau\right.$

$-\frac{413}{32} M_{1} \sin 3 \tau+\left(\frac{23,737}{32 \cdot 9 \cdot 25}+\frac{63}{256} M_{2}\right) \sin 4 \tau+\frac{75}{128} M_{1} \sin 5 \tau$

$\left.+\frac{3715}{2048 \cdot 3} \sin 6 \tau\right] m^{6}+\left[-\left(\frac{2,190,245}{1024 \cdot 3} M_{1}+\frac{105}{64} M_{3}\right) \sin \tau+\left(\frac{-578,077}{128 \cdot 243 \cdot 5}\right.\right.$

$\left.+\frac{34,155}{2048} M_{1}^{2}+\frac{5}{4} M_{2}\right) \sin 2 \tau-\frac{100,995}{2048} M_{1} \sin 3 \tau+\left(\frac{1,339,541}{128 \cdot 27 \cdot 125}\right.$

$\left.\left.-\frac{1725}{2048} M_{1}^{2}+\frac{77}{32 \cdot 3 \cdot 5} M_{2}\right) \sin 4 \tau-\frac{919}{1024} M_{1} \sin 5 \tau+\frac{4289}{16 \cdot 3 \cdot 5 \cdot 7} \sin 60\right] m^{7}$

$+\left[-\left(\frac{90,908,791}{8192 \cdot 3} M_{1}+\frac{1125}{128} M_{1} M_{2}+\frac{135}{16} M_{3}\right) \sin \tau-\left(\frac{54,988,283}{256 \cdot 729 \cdot 25}\right.\right.$

$\left.-\frac{811,967}{8192} M_{1}^{2}+\frac{45,721}{2048 \cdot 3} M_{2}\right) \sin 2 \tau-\left(\frac{7,378,645}{4096 \cdot 9} M_{1}-\frac{385}{512 \cdot 3} M_{3}\right) \sin 3 \tau$

$+\left(\frac{652,081,583}{8192 \cdot 81 \cdot 625}-\frac{10,125}{4096} M_{1}^{2}+\frac{91,069}{512 \cdot 9 \cdot 25} M_{2}\right) \sin 4 \tau-\left(\frac{133,763}{8192} M_{1}\right.$

$\left.-\frac{399}{512 \cdot 5} M_{3}\right) \sin 5 \tau+\left(\frac{338,729}{256 \cdot 5 \cdot 49}+\frac{1675}{16384} M_{1}^{2}+\frac{693}{2048} M_{2}\right) \sin 6 \tau$

$\left.+\frac{279,055}{8192 \cdot 49} M_{1} \sin 7 \tau+\frac{207,155}{131072 \cdot 3} \sin 8 \tau\right] m^{8}+\left[\left(-\frac{15,772,500,205}{32768 \cdot 27} M_{1}\right.\right.$

$\left.-\frac{17,415}{256} M_{1} M_{2}-\frac{84,575}{1024 \cdot 3} M_{3}-\frac{173,625}{16384} M_{1}^{3}\right) \sin \tau+\left(-\frac{1,601,782,811}{256 \cdot 2187 \cdot 125}\right.$

$\left.+\frac{4198}{9} M_{1}^{2}-\frac{182,881}{512 \cdot 3 \cdot 5} M_{2}\right) \sin 2 \tau+\left(-\frac{537,179,239}{4096 \cdot 27 \cdot 5} M_{1}+\frac{6075}{4096} M_{1} M_{2}\right.$

$\left.-\frac{9205}{2048 \cdot 3} M_{3}-\frac{14,625}{16384} M_{1}^{3}\right) \sin 3 \tau+\left(-\frac{3,297,305,351}{512 \cdot 81 \cdot 3125 \cdot 7}\right.$

$\left.+\frac{5,397,281}{32768 \cdot 9} M_{1}^{2}+\frac{1,097,537}{128 \cdot 27 \cdot 125} M_{2}\right) \sin 4 \tau+\left(-\frac{22,567,883}{32768 \cdot 9} M_{1}\right.$

$\left.-\frac{2205}{4096} M_{1} M_{2}+\frac{161}{2048} M_{3}\right) \sin 5 \tau+\left(\frac{1,673,061,443}{64 \cdot 81 \cdot 125 \cdot 343}-\frac{195,865}{16384 \cdot 7} M_{1}^{2}\right.$

$\left.+\frac{5659}{512 \cdot 3 \cdot 5} M_{2}\right) \sin 6 \tau-\frac{6857}{8192 \cdot 3 \cdot 7} M_{1} \sin 7 \tau$

$\left.+\frac{70,605.533}{32768 \cdot 3 \cdot 5 \cdot 49} \sin 8 \tau\right] m^{9}+\cdots$ 
If in these series $\eta$ is put equal to unity the terms which are independent of $M_{1}, M_{2}$, and $M_{3}$ correspond to those found by HILL in his researches on the Lunar Theory.* It is not easy to compare this work with HILL's for he used rectangular coördinates, and in the final series a different parameter. Moreover. his linear constant was different from the $a$ defined in (4) and used here. With $\eta=1$ the terms involving $M_{1}, M_{2}$, and $M_{3}$ correspond to those found by Brown. $\dagger$ The difficulty of comparing with his results is the same as that of comparing with Hill's work.

Delaunay used the reciprocal of the radius vector, the longitude, and the latitude as coördinates in his work on the Lunar Theory. Instead of the parameter $m$ he used in its place the ratio of the mean motion of the sun to that of the moon. If we call it $\mu$, it is

$$
\mu=\frac{n^{\prime}}{n}, \text { whence } m=\frac{\mu}{1-\mu} .
$$

Delaunay and other investigators in the Lunar Theory have used Fourier series whose coefficients are power series in the parameters employed. If we rearrange the series above as Fourier series and transform from $m$ to $\mu$, we may easily compare with DelaunaY's expression for the longitude. In order to make the comparison we must, in the series above, put

$$
\eta=1, \quad \frac{E^{j}-(-1)^{j} M^{j}}{(E+M)^{j}}=1, \quad m^{2 j} M_{j}=\left(\frac{a}{a^{\prime}}\right)^{j}
$$

The $\sin j \tau$ above is $\sin j \mathrm{D}$ in Delaunay's notation. Perfect agreement is found in the coefficients of all terms except the following:

* American Journal of Mathematics, vol. 1, p. 143.

† American Journal of Mathematics, vol. 14, pp. 140-160. 


\begin{tabular}{|c|c|c|}
\hline Coefficient of & Delaunay's value. & Present computation. \\
\hline & $63,106,813$ & $74,009,397$ \\
\hline$\frac{\omega}{a^{\prime}} \mu^{6} \sin \tau$ & $-\frac{00,100,010}{8192}$ & $-\frac{x, 000,}{8192}$ \\
\hline & $10,835,537,159$ & $47,823,726,517$ \\
\hline$\frac{\omega}{a^{\prime}} \mu^{7} \sin \tau$ & $-\frac{15}{65536 \cdot 3}$ & $-\frac{32768 \cdot 27}{3}$ \\
\hline$a)^{3}{ }^{2}$ & & 2415 \\
\hline$\left.\overline{a^{\prime}}\right)^{\mu^{2} \sin \tau}$ & not computed & $-\overline{128}$ \\
\hline$a)^{3} u^{3} \sin \tau$ & 66 & $\begin{array}{r}6,981,835 \\
\end{array}$ \\
\hline$a^{\prime} / \mu$ SIII & & $-\overline{16384 \cdot 3}$ \\
\hline & $7,596,606,727$ & $3,748,175,501$ \\
\hline$\mu^{\delta} \sin 2 \tau$ & $+\overline{4096 \cdot 729 \cdot 25}$ & $+\overline{2048 \cdot 729 \cdot 25}$ \\
\hline & $8,051,418,161$ & $475,281,673$ \\
\hline$\mu^{\jmath} \sin 2 \tau$ & $-\overline{2048 \cdot 2187 \cdot 125}$ & $-\overline{128 \cdot 2187 \cdot 125}$ \\
\hline$\left.\frac{a}{a^{\prime}}\right)^{2} \mu^{4} \sin 2 \tau$ & not computed & $+\frac{3,713,357}{8192 \cdot 3}$ \\
\hline$\left.\frac{a}{a^{\prime}}\right)^{2} \mu^{5} \sin 2 \tau$ & "6 & $+\frac{85,194,253}{2048 \cdot 9 \cdot 5}$ \\
\hline$\frac{a}{a^{\prime}} \mu^{6} \sin 3 \tau$ & 66 & $-\frac{21,715,555}{4096 \cdot 9}$ \\
\hline$\frac{a}{\mu^{7}} \mu^{7} \sin 3 \tau$ & 6 & - 1,764,511,039 \\
\hline$a$ & & $\begin{array}{c}4096 \cdot 27 \cdot 5 \\
385\end{array}$ \\
\hline$\left.\frac{a}{a^{\prime}}\right) \mu^{2} \sin 3 \tau$ & 6 & $+\frac{380}{512 \cdot 3}$ \\
\hline$\left.\frac{a}{a^{\prime}}\right)^{3} \mu^{3} \sin 3 \tau$ & 666 & $-\frac{19,975}{16384 \cdot 3}$ \\
\hline$\mu^{8} \sin 4 \tau$ & $+\frac{123,030,377,303}{8192 \cdot 81 \cdot 625}$ & $+\frac{82,679,012,303}{8192 \cdot 81 \cdot 625}$ \\
\hline$\mu^{9} \sin 4 \tau$ & not computed & $+\frac{876,528,810,303}{1024 \cdot 81 \cdot 3125 \cdot 7}$ \\
\hline$\left.\frac{a}{a^{\prime}}\right)^{2} \mu^{4} \sin 4 \tau$ & 6 & $-2,754.403$ \\
\hline$a)^{2} \sin 4 \pi$ & 66 & $\begin{array}{c}4096 \cdot 9 \cdot 25 \\
1,217,563,507 \\
\end{array}$ \\
\hline$\left.\overline{a^{\prime}}\right)^{\mu^{5} \sin 4 \tau}$ & 66 & $-\overline{32768 \cdot 27 \cdot 125}$ \\
\hline$a$ & 3911 & 1481 \\
\hline${\overline{a^{\prime}}}^{\mu^{5} \sin 5 \tau}$ & $+\overline{1024}$ & $+\overline{1024}$ \\
\hline$\frac{a}{a^{\prime}} \mu^{6} \sin 5 \tau$ & not computed & $-\frac{122,523}{8192}$ \\
\hline$\frac{a}{a^{\prime}} \mu^{7} \sin 5 \tau$ & 6 & $-\frac{51,974,771}{32768 \cdot 9}$ \\
\hline
\end{tabular}




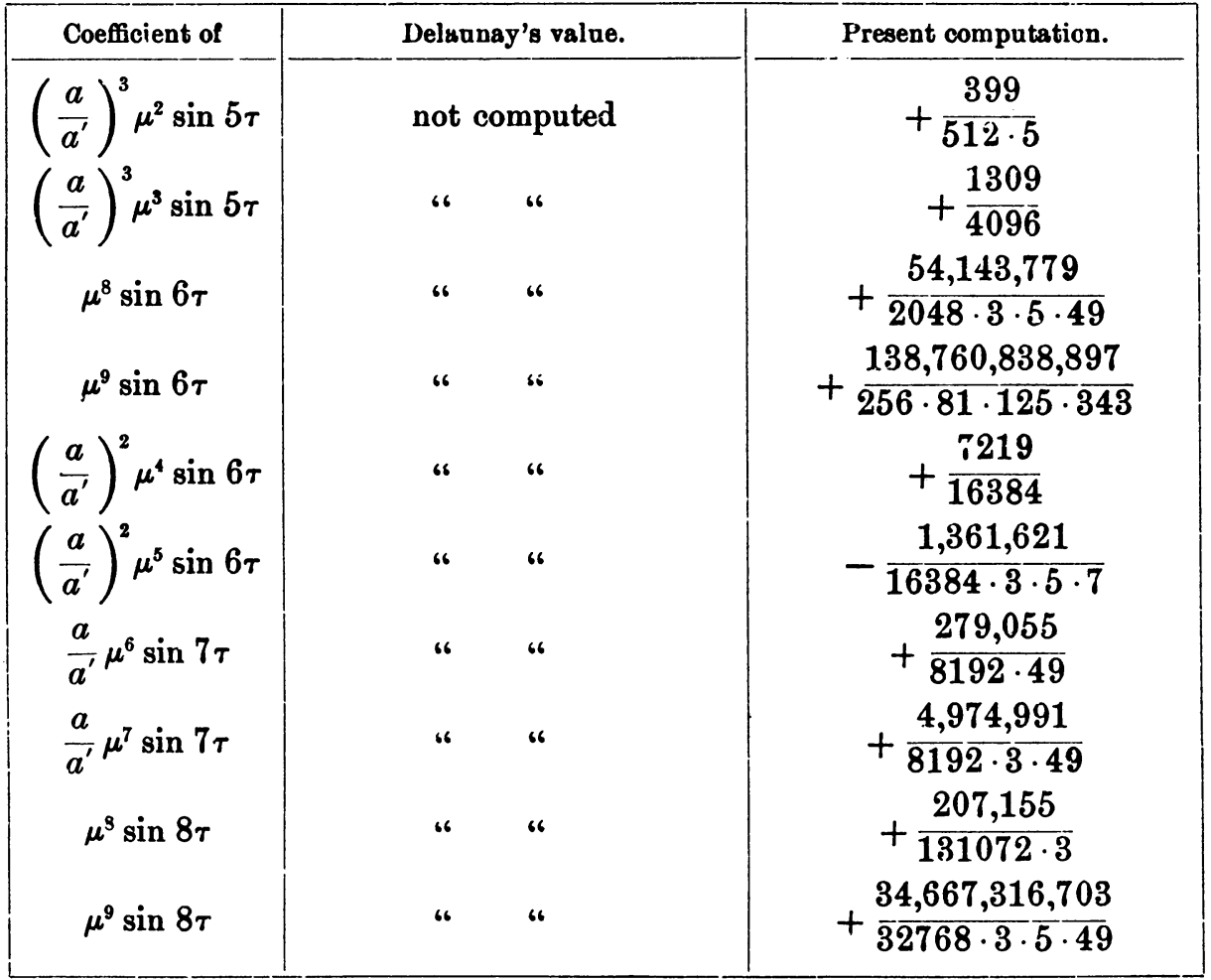

The very general agreement shows that Delaunay's terms involving the argument $j \mathrm{D}$ belong strictly to a periodic orbit, and that he carried out the computations with remarkable accuracy* considering the complicated operations which his method demanded.

When the expressions for the coördinates are arranged as Fourier series, it is found that the coefficients expressed in terms of $m$ converge much more rapidly, at least to the ninth power, than when expressed in terms of $\mu$. HiLL showed $\dagger$ that if a parameter $m^{\prime}$ is defined by

$$
m^{\prime}=\frac{m}{1-\frac{m}{3}},
$$

the convergence is still more improved. This transformation removes the pole arising in the expansion of the principal term from $|m|=\sqrt{6}$ to $\left|m^{\prime}\right|=3$. In $\mu$ the pole is at the distance $|\mu|=\sqrt{\frac{6}{11}}$. If an $m^{\prime \prime}$ is introduced by

* Brown's results disagree with DerlaUnay's in the coefficients of $\left(a ! a^{\prime}\right) \mu^{5} \sin j \mathrm{D}$; he did not compute higher powers. Loc. cit., p. 155.

$\ddagger$ Loc. oit., p. 141. 


$$
m^{\prime \prime}=\frac{m^{2}}{6-4 m+m^{2}}
$$

the pole of the expression is removed to infinity and the convergence of the series for the coefficients of $\cos 2 \tau$ and $\sin 2 \tau$, at least out to the 9 th power, is slightly improved. But these coefficients are given by any of the series with sufficient accuracy. The slowest convergence is in the coefficient of $\sin \tau$. In Delaunay's parameter this coefficient is

$$
\begin{gathered}
{\left[-\frac{15}{8} \mu-\frac{93}{8} \mu^{2}-\frac{6887}{128} \mu^{3}-\frac{137,197}{512} \mu^{4}-\frac{4,628,333}{1024 \cdot 3} \mu^{5}-\frac{74,009,397}{8192} \mu^{6}\right.} \\
\left.\frac{-47,823,726,517}{32768 \cdot 27} \mu^{7} \cdots\right] \frac{a}{a^{\prime}} .
\end{gathered}
$$

In the parameter of this paper the same coefficient is

$$
\begin{array}{r}
{\left[-\frac{15}{8} m-\frac{39}{4} m^{2}-\frac{4151}{128} m^{3}-\frac{71,449}{512} m^{4}-\frac{2,190,245}{1024 \cdot 3} m^{5}-\frac{90,908,791}{8192 \cdot 3} m^{6}\right.} \\
\left.-\frac{15,772,500,205}{32768 \cdot 27} m^{7} \cdots\right] \frac{a}{a^{\prime}} .
\end{array}
$$

When expressed in seconds the coefficient of the term containing the 6 th and 7 th powers of the parameter is $-0^{\prime \prime} .7122$ and $-0^{\prime \prime} .3811$ in Delaunay's series, and $-0^{\prime \prime} .5453$ and $-0^{\prime \prime} .2124$ in the series given here. While the two series represent the same function, the first seven terms in $m$ seem to give it more accurately than the first seven terms in $\mu$.

It is noticed that the numerical coefficients so far as given are all of the same sign, and that the ratio of each coefficient to the preceding one seems to tend approximately toward 5 . If this were the precise ratio, and if it were indefinitely maintained, the series would be simply the expansion of $(1-5 m)^{-1}$. If the first few terms succeeding those which have been computed proceed approximately according to the samie law, then the function will be much more accurately given by them if they are multiplied and divided by $1-5 m$. Thus we find by these operations in place of $(52)$

$$
\left[\begin{array}{r}
-\frac{15}{8} m-\frac{3}{8} m^{2}+\frac{2089}{128} m^{3}+\frac{11,571}{512} m^{4}-\frac{46,775}{1024 \cdot 3} m^{5}-\frac{3,298,991}{3 \cdot 81 \overline{92}} m^{6} \\
+\frac{591,082,175}{32768 \cdot 27}-m^{7} \cdots
\end{array}\right] \frac{a}{a^{\prime \prime}}
$$

In applying HrLL's method to the parallactic inequalities BRown found * that

\footnotetext{
* Loo. oit., p. 149.
} 
the divisor $1-4 m-37 m^{2} \cdots$ naturally enters. The terms in the series with this divisor decrease somewhat less rapidly than those in (53). It is to be noted that the radius of convergence is never altered by using any finite polynomial as a multiplier and divisor in this way. The process is in reality a sort of method of exterpolating which is useful if the coefficients proceed for a time according to the assumed law.

The average ratio of each coefficient to the preceding one for the six terms given in (52) is approximately $\frac{23}{5}$. Hence we may conveniently write the series as $-\frac{15}{8} m\left[1-2 \frac{2}{5}-m\right]^{-1}$ plus a series which shall make, when added to the expansion of this function, the six terms given in (52). We find in place of (52)

$$
\begin{aligned}
& {\left[-\frac{15}{3} m\left[1-\frac{23}{5} m\right]^{-1}-\frac{9}{8} m^{2}+\frac{4637}{128 \cdot 5} m^{3}+\frac{549,839}{512 \cdot 25} m^{4}\right.} \\
& \left.+\frac{48,596,207}{1024 \cdot 3 \cdot 125} m^{5}+\frac{2,499,342,713}{8192 \cdot 3 \cdot 625} m^{6}-\frac{174,308,031,761}{32768 \cdot 27 \cdot 3125} m^{7}+\cdots\right] \frac{a}{a^{\prime}}
\end{aligned}
$$

To illustrate the effects of these processes we shall reduce these series to numbers. It has been found from the observations that

$$
m=0.08084893, \quad \frac{a}{\iota^{\prime}}=0.00255878 .
$$

With these numbers we get for (52), (53), and (54) respectively

$-80^{\prime \prime} .008-33^{\prime \prime} .637-9^{\prime \prime} .045-3^{\prime \prime} .147-1^{\prime \prime} .300-0 " .545$

$$
-0^{\prime \prime} .212 \cdots=-127^{\prime \prime} .894 \text {, }
$$

$\frac{-80^{\prime \prime} 008-1^{\prime \prime} .294+4^{\prime \prime} .552+0^{\prime \prime} .516-0^{\prime \prime} .028-0^{\prime \prime} .020+0^{\prime \prime} .008 \ldots}{0.59575535}$

$$
=-128^{\prime \prime} .029
$$

$-127^{\prime \prime} .382-3^{\prime \prime} .882+2^{\prime \prime} .021+0^{\prime \prime} .969+0^{\prime \prime} .231+0^{\prime \prime} .024$

$$
-0^{\prime \prime} .001 \cdots=-128^{\prime \prime} .020 \text {. }
$$

The corresponding values in DelaunaY's series including a term of the 7 th degree in $\mu$ are

$$
\begin{aligned}
-74^{\prime \prime} .024-34^{\prime \prime} .330-11^{\prime \prime} .885-4^{\prime \prime} .428-1^{\prime \prime} .862 \\
-0^{\prime \prime} .712-0^{\prime \prime} .381 \cdots=-127^{\prime \prime} .622 .
\end{aligned}
$$

Brown found ${ }^{*}$ for this coefficient, using the numerical value of $m$ from the outset, $-128^{\prime \prime} .069$. While this number is slightly larger than one would infer

* Monthly Notices of R. A. S., vol. 64 (1904), 534. 
from (52), (53), or (54), it is -very probably essentially correct. But in the computation we put $\eta=1, M_{1} m^{2}=a / a^{\prime}$, whereas the correct values are

$$
\begin{gathered}
\eta=\frac{1}{1+\frac{E+M}{S^{\prime}}}=0.999997, \\
M_{1} m^{2}=\frac{E-M}{E+M} \frac{a}{a^{\prime}}=\frac{80.487}{82.487} \frac{a}{a^{\prime}} .
\end{gathered}
$$

With these values of the constants the coefficient (53) is $-124^{\prime \prime} .925$. Hill has computed $\dagger$ the coefficients of the various arguments of the series with a very high degree of accuracy by substituting their approximate values as determined by himself and Brown in the differential equations, and giving them such differential corrections that they more exactly satisfy the equations. He found for the coefficient of $\sin \tau$, when we change from his value of $a / a^{\prime}$ to that used here, $-124^{\prime \prime} .965$.

\section{Applications to Darwin's Periodic orbits.}

In his memoir in Acta Mathematica, vol. 21, pp. 99-242, DARwIN considered two finite bodies of masses 10 and 1 revolving around their center of gravity in circles, and an infinitesimal body moving in the plane of motion of the finite bodies and subject only to their attraction. He discovered a large number of periodic orbits by interpolation from series of special orbits computed by mechanical quadratures. It will be shown that his "Satellites $A$ " and "Planets $A$ " belong to the type of orbits treated in this paper.

To make the series computed in this paper applicable to the satellite orbits treated by DARwiN it is necessary to put $M=0, E=1, S=10, a^{\prime}=1$. Then, when the infinitesimal body moves around $E$, we have from (4) and (6),

$$
\begin{aligned}
\eta & =\frac{S}{E+S}=\frac{10}{11}, \\
\frac{a}{a^{\prime}} & =\left(\frac{n^{\prime}}{n}\right)^{\frac{3}{3}}\left(\begin{array}{c}
E \\
E+S
\end{array}\right)^{\frac{1}{3}}=\left(\frac{1}{11}\right)^{\frac{1}{3}}\left(\frac{m}{1+m}\right)^{\frac{3}{3}} .
\end{aligned}
$$

DARwIN defined his orbits by the value of the Jacobian constant, and their periods were found from the detailed computations. The Jacobian integral may be written

$$
F=F_{0}+F_{1} m+F_{2} m^{2}+\cdots=C .
$$

For an arbitrary value of $m$ sufficiently small, as $m_{0}$, the value of $C$ can be computed. Let it be $C_{0}$; then

*Astronomical Journal, vol. 15 (189), p. 137.

Trans. Am. Math. Soc. 38 


$$
F_{0}+F_{1} m_{0}+F_{2} m_{0}^{2}+\cdots=C_{0} .
$$

Let the value of $C$ of the required orbit be $C=C_{0}+\Delta C$, and the corresponding value of $m$ be $m=m_{0}+\Delta m$. Substituting these values in the integral and inverting the series, we have $\Delta m$ expressed in terms of $\Delta C$, and therefore $m$ expressed in terms of $C$. With this value of $m$ the coördinates can be computed from the series which have been given above.

While there is no difficulty in carrying out the details of the steps just described, it will be sufficient for the comparison to take the period as found by the computation. We shall compare with "Satellite $A$ " (Jacobian constant $=40.5$ ), loc. cit. p. 199. The synodic period was found to be $61^{\circ} 23^{\prime}=61^{\circ} .383$, where the period of the finite bodies is $360^{\circ}$. Therefore

$$
\begin{gathered}
m=\frac{61.383}{360}=\frac{1}{5.8648}=0.17051, \\
\frac{a}{a^{\prime}}=\left(\frac{1}{11}\right)^{t}\left(\frac{m}{1+m}\right)^{3}=0.12449 .
\end{gathered}
$$

The $m$ is more than twice as large as that occurring in the Lunar Theory, while the $a / a^{\prime}$ is more than $\mathbf{4 0}$ times as great as $a / a^{\prime}$ for the moon and sun.

Putting $M_{j} m^{2 j}=\left(a / a^{\prime}\right)^{j}$ in the series, and using these values of the constants we find

$$
\begin{aligned}
r=0.12427+0.00652 \cos \tau-0.00420 \cos 2 \tau+0.00004 \cos 3 \tau & \\
& \quad-0.00006 \cos 4 \tau \ldots, \\
w=-0.12062 \sin \tau+0.05079 \sin 2 \tau-0.00184 \sin 3 \tau & +0.00095 \sin 4 \tau \ldots
\end{aligned}
$$

The infinitesimal body is in a line with the finite bodies and between them when $\tau=0$. The value of $r$ at this time is $r(0)=0.12657$. The corresponding value given by DARwIN is 0.1265 , an agreement within the limits of accuracy of his computations. The infinitesimal body is in opposition at $\tau=\pi$, and we find for the radius at this time $r(\pi)=0.11345$. DARwis's value is 0.1135 . The agreements are sufficient to show that the analysis of this paper pertains to DARwiv's "Satellites $A$."

To get the coördinates in a retrograde orbit having the same sidereal period, it is necessary to change the sign of $n$ in $m=n^{\prime} /\left(n-n^{\prime}\right)$. In the example just treated it is found that

$$
\frac{n^{\prime}}{n}=\frac{61.383}{421,383}
$$

whence $m$ in the retrograde orbit having the same sidereal period is 


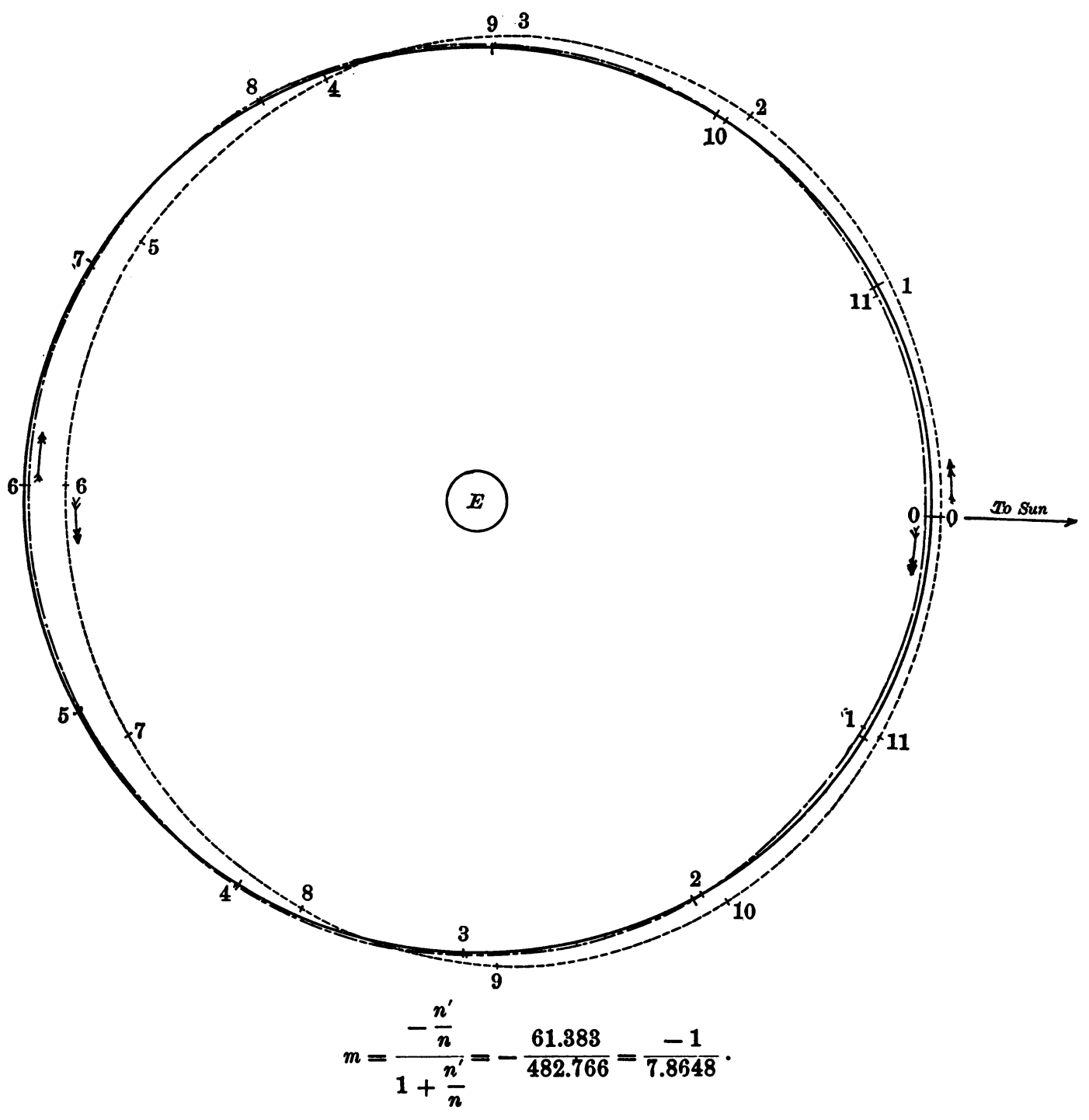

The value of $a / a^{\prime}$ is the same as before and the series give for the coördinates $r=+0.12412-0.00057 \cos \tau-0.00158 \cos 2 \tau$ $-0.00005 \cos 8 \tau-0.00001 \cos 4 \tau+\cdots$, 
$w=+0.00820 \sin \tau+0.01654 \sin 2 \tau+0.00047 \sin 3 \tau+0.00010 \sin 4 \tau+\cdots$

These two orbits and the circular orbit of which they are the analytic continuation, together with the positions at intervals of $\frac{1}{12}$ of the period, are given in the figure.

DARwIN found periodic planetary orbits corresponding to the "Satellites $A$." The analysis above gives these orbits within the limits of the convergence of the series if we put $E=10, S=1, M=0, a^{\prime}=1, \eta=S /\left(E+S^{\prime}\right)=\frac{1}{11}$. For the comparison with an actual example we shall take "Planet $A$ " for the Jacobian constant equal to 40.0 (loc. cit., p. 225). The period given by DARwis's computation is $n T=154^{\circ} 13^{\prime}$, whence

$$
m=\frac{154.216}{360}=\frac{1}{2.3344}=0.42838, \quad \frac{a}{a^{\prime}}=0.43404, \quad M_{1}=2.3652 .
$$

With these values of the parameters the series for the radius becomes

$$
r=0.43373+0.00776 \cos \tau-0.01286 \cos 2 \tau-0.00104 \cos 3 \tau \cdots
$$

When the infinitesimal body is between $E$ and $S$, and when it is in opposition to $S$, the values of $r$ are respectively $r=0.42759$ and $r=0.41415$. DARwiN found in these respective cases $r=0.423$ and $r=0.4140$. These results agree within the limits of error of the computations and show that the "Planets $A$ " belong to the type of orbits covered by the analysis of this paper.

DARwiN's "Satellites $B$ " and "Satellites $C$ " do not exist until the Jacobian constant has diminished nearly to 39.5. At first appearance they are coincident, but with decreasing values of the Jacobian constant they gradually diverge. The computations of DARwiN show that they are not isolated orbits existing only for special values of the parameter $m$. Suppose one of these orbits is known for $m=m_{0}$. Unless the orbits " $B$ " and " $C$ " coincide for this value of $m$, the coördinates of an orbit near the known one may be expressed as power series in $m-m_{0}=\lambda$; if the orbits " $B$ " and " $C$ " coincide for this value of $m$, the coördinates of the two neighboring orbits may be expressed as power series in $\sqrt{\lambda}$ and $-\sqrt{\lambda}$ respectively. When $\lambda$ has one sign their coördinates are real, and when it has the other, they are conjugate complex quantities. Suppose the series converge so long as $|\lambda|<\lambda_{0}$. The coördinates of the orbits near the ones belonging to $m=m_{0}+\lambda$ may be expanded as power series in $m-m_{0}-\lambda_{0}=\lambda^{\prime}$. If the complex orbits are in question there will be two, one with $\lambda$ and one with $-\lambda$, whose coördinates will still be complex and conjugate. They have the property of reëntering after one synodic revolution.

Suppose the two orbits which branch at $m=m_{0}$ are followed until $m_{0}+\lambda_{0}+\cdots=0$. Their coördinates will remain conjugate through the whole series of steps, and the property of reëntering after one synodic revolution will 
be preserved. But for this value of $m$ the disturbance vanishes and the differential equations reduce to those which belong to the two body problem. The period remains real in $t$ as well as in $\tau$. Consequently these orbits show that there are at least two conjugate complex solutions of the problem of two bodies whose period in $t$ is real.

Let us consider the differential equations expressed in $t$. From (6) we have

$$
\tau=\frac{n^{\prime}}{m} t=n\left(1-\frac{n^{\prime}}{n}\right) t,
$$

and the differential equations may be expressed in terms of $n^{\prime} / n=\mu$. The parameter enters in two ways, $(a)$ independently of the trigonometric functions, and (b) under the trigonometric functions. We shall generalize the differential equations by distinguishing the $\mu$ according as it enters in one way or the other. Where it enters under the trigonometric functions it will be regarded as an assigned number which admits of no change in the development of the solutions; where it enters in the other way it will be regarded as a parameter capable of taking all values sufficiently small, and it will be made to play the same rôle as $m$ has in the preceding work. The solutions exist for all values of $\mu$ sufficiently small, but they belong to the physical problem only when the parameter $\mu$ is set equal to the $\mu$ which occurs under the trigonometrical functions. The series of orbits is obtained by assigning a series of values to the $\mu$ in the trigonometric functions.

In the orbits in question the angular motion of the finite bodies is real; therefore $n^{\prime}$ is real. Also the period of the infinitesimal body is real ; therefore $\mu$ and $n$ are real. The orbit of the finite bodies is real; therefore $a^{\prime}$ is real and equal to unity with DARwin's choice of the linear unit. The parameter $a$ is subjected only to the relation (7)

$$
\omega=a^{\prime}\left(\frac{m}{1+n}\right)^{3}\left(\frac{E}{E+S}\right)^{3} \quad(M \text { being infinitesimal }) .
$$

There are precisely three values of $a$ satisfying this relation, one of which is real while the other two are conjugate complex quantities. All three satisfy the conditions of the two body problem equally. Likewise the periodic solutions of the three body problem exist for all three roots for all values of $\mu$ sufficiently small. Moreover these are the only solutions of the type under consideration in which the periods are real. In the three cases the coefficients of all powers of $a / a^{\prime}$ are precisely the same, and since the three values of $a / a^{\prime}$ have the same modulus, the series have the same radius of convergence. Since these are the only solutions of the type in question, it follows that DaRwin's orbits "Satellites $B$ " and "Satellites $C$ " are the analytic continuation of these complex orbits, and that his search for the satellite orbits was exhaustive. His work 
shows the interesting and important result that the two imaginary orbits unite and become real (if the distribution of masses is 10 and 1 ) when $m$ is a little less than 1 .

The remaining problem respecting the series which have been developed is their true radius of convergence for a given distribution of mass between the finite bodies. For simplicity suppose the coördinates and components of velocity of the infinitesimal body are $x_{1}, \ldots, x_{4}$. Then the differential equations may be written

$$
\frac{d x_{j}}{d t}=f_{j}\left(x_{1}, \ldots, x_{4}, \mu ; t\right) \quad(j=1, \cdots, 4) .
$$

The initial values of the $x_{j}$ are $\alpha, e, 0,0$. Then the solutions may be written

$$
x_{j}=p_{j}(\alpha, e, \mu ; t),
$$

which converge for any preassigned interval in $t$ if the moduli of $\alpha, e$ and $m$ are sufficiently small. HAPPEL* has shown that they converge for the values of these parameters which belong to the moon's variational orbit. But this by no means proves the convergence of the series in $\mu$ which belong to the variational orbit. The parameters $\alpha$ and $e$ are determined in terms of $\mu$ by the periodicity conditions (on two of the equations for symmetrical orbits)

$$
p_{i}\left(\alpha, e, \mu ; \frac{T}{2}\right)=0, \quad p_{j}\left(\alpha, e, \mu ; \frac{T}{2}\right)=0,
$$

where $T$ is the period. These series are to be solved for $\alpha$ and $e$ as power series in $\mu$ and the results substituted in (56). In general, new singularities are introduced by the conditions (57) and are carried back into (56). Consequently in discussing the convergency of the series for the variational orbit both the series $(56)$ and the singularities introduced by (57) must be considered.

Consider equations (57). For that value of $\mu$ for which two orbits coincide these equations have a double solution, and this value of $\mu$ is a branch point of their solutions. Therefore the periodic solutions in $\mu$ do not converge for values of $\mu$ whose moduli are greater than the modulus of that value of $\mu$ for which two of the orbits coincide. Applying this to Darwin's computations, we find that the series for the complex orbits " $B$ " and " $C$ " do not converge for values of $\mu$ beyond which these orbits unite and become real. The series for the orbit " $A$ " have the same radius of convergence as those for the complex orbits. Therefore the series for " $A$ " do not converge for values of $\mu$ greater than that for which " $B$ " and " $C$ " become real. Expressed in $m$, the series do not converge for $|m|>\frac{1}{4}$. In the orbit computed $m=1 / 5.86$, and an examination of the coefficients shows that this is near the limit of practical useful-

* Inaugural Dissertation, Göttingen, 1900. 
ness of the series. Many of Darwin's orbits belong to values of $m$ for which the series diverge.

Unfortunately it cannot be positively asserted that the series converge until the orbits " $B$ " and " $C$ " become real. In the first place the parameter $\mu$ must be considered as complex except as it appears under the trigonometric functions, where it must remain real. (The radius of convergence is entirely independent of the value of the parameter under the sine and cosine functions.) Now it might happen that there would be a branching of orbits for a smaller modulus of $\mu$ in this complex realm. Such computations as those made by DARwiN would fail to show it, for the orbits, real or imaginary, belong to the physical problem only when $\mu$, as the argument of the series, has the same value as occurs under the trigonometric functions.

The functions may branch in another way. An orbit which in general reënters only after two synodic revolutions may for a special value of $\mu$ coincide with one which reënters after one synodic revolution. At this point the orbit under consideration changes its stability. * DARwis's "Satellite $A$ " loses its stability for $m$ about 1/3.7. However, this seems not to have been because of a branching of the character in question, but because of a union of the orbit with the orbit of an "Oscillating Satellite $a . " *$ But there is no earlier change of stability, at least for real values of $\mu$.

The series do not have a fixed radius of convergence as $t$ goes through a period. The convergence in question is a uniform convergence with respect to $t$ over the period of motion, and in this sense we have spoken of the series as having radii of convergence.

The problem of chief practical interest is the Lunar Theory. The distribution of mass between the finite bodies assumed by DARwin, as compared with that occurring in the Lunar Theory, has decreased $\eta$ a little and has increased $a / a^{\prime}$ for a given value of $m$ about 30 fold. It seems probable that in the Lunar Theory the series converge for larger values of $m$ than in DARwin's problem, and the computation seems to show that in the latter problem they converge for $m=\frac{1}{6}$. Although it can scarcely be doubted that the series converge for the values of the parameters which the Lunar Theory requires, a proof of their convergence for these values is yet wanting. However, we do know that there is a limit within which the series are perfectly valid.

The University of Chicago.

* Poincaré's Les Méthodes Noltvelles, vol. 3, art. 380. 